\title{
Breeding for Earliness and Seed Yield in Summer Sesame (Sesamum indicum L.) Over Environments
}

\author{
R.S. Parmar ${ }^{1 *}$, V.P. Chovatia ${ }^{2}$, G.K. Sapara ${ }^{3}$ and J.C. Dhingani ${ }^{3}$ \\ ${ }^{1}$ College of Agriculture, Junagadh Agricultural University, Motabhandariya (Amreli)-355610, \\ Gujarat, India \\ ${ }^{2}$ Director of Research and P.G. Studies, Junagadh Agricultural University, Junagadh, \\ Gujarat, India \\ ${ }^{3}$ Main Oilseeds Research Station, JAU, Junagadh, Gujarat, India \\ *Corresponding author
}

\begin{abstract}
A B S T R A C T
Sesame is an important oilseed crop not only in India also across the world. Studying nature of inheritance of sesame plant characteristics is essential for planning effective

Keywords

Sesame, Seed yield, Earliness and heteroiss

\section{Article Info}

Accepted:

12 September 2018

Available Online:

10 October 2018

breeding programme. The present investigation was conducted at the Agricultural Research Station, J.A.U., Amreli and Department of Seed Science and Technology, College of Agriculture, Sagadividi Farm, J.A.U., Junagadh, Gujarat. These 40 hybrids along with a two checks, G.Til-2 and G.Til-3 were evaluated in a four environments were created through different time and location for sowing during summer 2016. The earliest hybrid over better parent was AT-306 x RT-54, AT-322 x RT-54, AT-307 x RT-54, AT307 x G.Til-10 and AT-253 x RT-54 in days to flowering in pooled. The crosses AT-306 x AT-285 and AT-306 x AT-285 recorded the highest desirable standard heterosis over G.Til-2 and G.Til-3 in days to maturity. The best hybrids AT-319 x AT-285, AT-322 x AT-285, AT-307 x AT-285, AT-319 x G.Til-10 and AT-306 x AT-285 exhibited significant and positive heterobeltiosis for seed yield per plant and its components. Such crosses could be exploited further for earliness and seed yield advancement in sesame through regional multilocational testing over different environments.
\end{abstract}

\section{Introduction}

Sesame (Sesamum indicum L.), commonly known as gingelly, til, simsim, til or tal is a member of the order Tubiflorae and family Pedaliaceae. Sesame is predominantly annual self-pollinated $(85-95 \%)$ diploid $(2 n=2 x=26)$ crop. It is referred as 'Queen of Oilseeds' due to its regard by the users and owing to its oil quality (Bedigian and Harlan, 1986). It is one of the most ancient crops in the world known to mankind, with archeological evidences dating back to 2250 and $1750 \mathrm{BC}$ at Harappa in the Indus valley (Najeeb et al., 2012). Its oil was extracted by the ancient Hindus, which was used for certain ritual purposes (Weiss, 1983). Ironically, it is considered as an 'orphan crop' due to meager research efforts attributed to the fact that it is not a mandate crop for any international crop research 
institute (Bhat et al., 1999). Sesame is an important annual oilseed crop in the tropics and warm subtropics. At present, Myanmar is the largest producer of sesame seed in the world followed by India, China, Ethiopia, Nigeria and Uganda. In India, during 2015-16, sesame is cultivated in an area of 17.46 lakh ha with a production of 9.11 lakh tones annually and productivity of $474 \mathrm{~kg} / \mathrm{ha}$ (Anon., 2016). Being the fourth important oilseed crop in Indian agriculture after groundnut, rape seed and mustard, it is widely cultivated in the states of Uttar Pradesh, Rajasthan, Orissa, Gujarat, Andhra Pradesh, Tamil Nadu, Karnataka, West Bengal, Bihar and Assam. In Gujarat, during 2015-16, sesame is cultivated in an area of 2.56 lakh ha with a production of 1.52 lakh tones and productivity of $530 \mathrm{~kg} / \mathrm{ha}$ (Anon., 2016). This crop is generally cultivated as sole or mixed crop during kharif, semi-rabi and summer season. The productivity of sesame is very low as compared to other oilseeds hence, it is necessary to raise the productivity and thereby total oilseeds production in order to meet edible oil requirement of the country.

The first important step in the exploitation of heterosis is to know its magnitude and direction. The nature and magnitude of heterosis help in identifying superior cross combinations and their exploitation to get better transgressive segregants. The theory of dominant linked gene hypothesis put forward by Jones (1917) and advocated and discussed in Singh and Singh (1984) appears to be the most acceptable, both in concept and utilization of hybrid vigour in self-pollinated crops. According to them one can select a pure breeding line equally good or even better than $\mathrm{F}_{1}$ hybrid.

In the present decade, considerable emphasis has been laid on the development of hybrids and high yielding varieties. For improving yield potential and to have a quantum jump in yield of varieties and hybrids, selection of right type of parents is very important. For this, the study of genetic parameters is the first requisite for better understanding of the genetic make-up of the genotypes especially in the systematic breeding programme because many times the high yielding genotypes may not combine well either to give a good cross combination or to give desirable segregants. Parents which produce good progenies on crossing are of immense use of the breeder. The necessities of testing the parents for their combining ability in turn will help in identifying the best combiners, which may be hybridized. Hybridization may aim to exploit heterosis or to accumulate fixable genes through selection in segregating generations. Thus, the knowledge of relative magnitude of additive and non-additive components of genetic variance and their exploitation to its maximum extent has become importance.

The computation of relative heterosis has no genetic basis and hence not estimated. Hence, heterosis over better parent (heterobeltiosis) and heterosis over standard check G.Til-2 and G.Til-3 (standard heterosis) with significance in the desirable direction have taken into consideration for comparing the different crosses in the present investigation. The aim of heterosis estimation in the present study has to spot out the best combination of parents giving high degree of useful heterosis and characterization of parents for their genotypic worth for future use in breeding programme. The degree and magnitude of heterosis varied from cross to cross for all the characters under individual environments and in pooled analysis.

Earliness characters are of paramount importance in breeding for early maturing varieties/hybrids of oilseed crops in general and sesame in particular for better adaptation to climate change (Paroda, 2013). Early maturing varieties contribute significantly to 
increasing productivity. Selection for maturation period can be effective using flowering period for improving uniform ripening capsule. A suitable breeding methodology and the identification of superior parents are the most important pre-requisites for the development of early maturing and high yielding genotypes. For genetic improvement of the crop, the breeding method to be adopted is depending upon the nature of gene action involved in the expression of different characters. The genetic makeup of genotypes for quantitatively inherited traits can be well understood by the study of genetic parameters. Breeding analysis has been utilized to know the nature and extent of gene action controlling expression of different characters including seed yield and would help in proper planning of a successful breeding programme.

\section{Materials and Methods}

The experimental material comprised of four females as testers viz., AT-285, G.Til-1, G.Til10 and RT-54 and ten males as lines namely AT-253, AT-265, AT-306, AT-307, AT-319, AT-322, AT-341, Bhuva-2, Khadkala-S and IS-209 and their 40 hybrids derived from line $\mathrm{x}$ tester mating design. These 56 genotypes along with a two checks, G.Til-2 and G.Til-3 were evaluated in a four environments were created through different time and location for sowing during summer 2016 i.e., $\mathrm{E}_{1}=$ Timely sowing $3^{\text {rd }}$ week of February at Amreli $\left(20^{\text {th }}\right.$ February), $\mathrm{E}_{2}=$ Timely sowing $3^{\text {rd }}$ week of February at Junagadh ( $20^{\text {th }}$ February), $\mathrm{E}_{3}=$ Late sowing $2^{\text {nd }}$ week of March at Amreli $\left(10^{\text {th }}\right.$ March), and $\mathrm{E}_{4}=$ Late sowing $2^{\text {nd }}$ week of March at Junagadh $\left(10^{\text {th }}\right.$ March $)$. The parents and $F_{1}$ 's with checks were sown in single row (plot) of $2.25 \mathrm{~m}$ length with spacing $45 \mathrm{~cm} \mathrm{x}$ $15 \mathrm{~cm}$. All the agronomical practices and plant protection measures were followed as and when required to raise a good crop of sesame. The observations were recorded on five randomly selected plants from parents and crosses for all characters viz, days to flowering, days to maturity, plant height $(\mathrm{cm})$, height to first capsule $(\mathrm{cm})$ and seed yield per plant (g).Agricultural Research Station, J.A.U., Amreli is located in North Saurashtra Agro-climatic zone-VI of Gujarat state. Deptt. Seed Science and Technology, Sagadividi Farm, J.A.U. Junagadh is located in South Saurashtra Agro Climatic Zone-III of Gujarat state. Standard procedures for analysis of variance were followed. Data were first subjected to the analysis followed for randomized block design as per Panse and Sukhatme (1967). Theoretically heterosis is the deviation of $F_{1}$ from the mid parental value. But, an increase in $F_{1}$ over poor parents may not be of practical importance. Therefore, in the present investigation, heterosis was estimated over better parent (BP), which is referred as heterobeltiosis as per Fonseca and Patterson (1968). In addition, an increase in $F_{1}$ over the standard check hybrid (standard heterosis) is of commercial importance. Hence, the standard heterosis was also worked-out by using popular two check G.Til2 and G.Til-3.

\section{Results and Discussion}

The pooled analysis of variance over environments (Table 1) showed significant differences among the environments (sowing dates and different locations) for all the characters indicating wide variation in environmental conditions or differential expression of traits under different sowing dates and different location. Parents and hybrids mean squares were significant for all the characters under all environments indicating the presence of sufficient diversity among the parents and hybrids for all the characters. The mean squares due to parents vs. hybrids contrast also revealed significant differences for all the characters under all the environments as well as pooled over 
environments except $\mathrm{P}$ vs. $\mathrm{H}$ for seed yield per plant in pooled over environments. Highly significant mean squares due to parents $\mathrm{x}$ environments, hybrids $\mathrm{x}$ environments, ( $\mathrm{P}$ vs. $\mathrm{H}) \mathrm{x} E$ were observed for all the traits for $(\mathrm{P}$ vs. H) x E mean squares. This emphasized that parents $\mathrm{X}$ environments, hybrids $\mathrm{X}$ environments and ( $\mathrm{P}$ vs. $\mathrm{H}) \mathrm{x} \mathrm{E}$ interacted differently to diverse environments and environments had noticeable influence on these traits. Mean squares due to hybrids were highly significant for all the characters studied. The performance of variance due to $\mathrm{P}$ vs $\mathrm{H}$ differed significantly than that of parents suggesting the presence of average heterosis for all the traits under all the environments except seed yield per plant in pooled over environments.

For earliness traits like days to flowering (Table 2) in which hybrid AT-306 x RT-54 in $\mathrm{E}_{1}$, AT-307 x G.Til-10 in $\mathrm{E}_{2}$, AT-307 x RT54in $\mathrm{E}_{3}$, AT-307 x G.Til-10 in $\mathrm{E}_{4}$; and AT-306 x RT-54 in pooled analysis recorded earliest. The earliest hybrid over better parent was AT$306 \times$ RT-54 followed by AT-322 x RT-54, AT-307 x RT-54, AT-307 x G.Til-10 and AT$253 \times$ RT-54. Out of 40 hybrids, significant and negative heterobeltiosis crosses was recorded in 9, 4, 32, 20 and 3 in $\mathrm{E}_{1}, \mathrm{E}_{2}, \mathrm{E}_{3}, \mathrm{E}_{4}$ and across the environments, respectively. The range of earliest standard heterosis $\left(\mathrm{H}_{2} \mathrm{C}_{1}\right)$ over G.Til-2 ranged from -8.33 to $6.67 \%$, 9.76 to $8.13 \%,-5.91$ to $16.19 \%,-8.62$ to 8.62 $\%$ and -6.47 to $6.90 \%$ in $\mathrm{E}_{1}, \mathrm{E}_{2}, \mathrm{E}_{3}, \mathrm{E}_{4}$ and pooled analysis, respectively. In case of G.Til3 the magnitude of standard heterosis $\left(\mathrm{H}_{2} \mathrm{C}_{2}\right)$ ranged from -4.35 to $11.30 \%,-7.50$ to 10.83 $\%,-11.61$ to $8.93 \%,-6.19$ to $11.50 \%$ and 5.65 to $7.83 \%$ in $\mathrm{E}_{1}, \mathrm{E}_{2}, \mathrm{E}_{3}, \mathrm{E}_{4}$ and pooled analysis, respectively.

In earliness traits like days to maturity (Table 3 ) the range of heterosis over heterobeltiosis was -4.91 to $10.89 \%,-5.28$ to $10.67 \%,-13.21$ to $-0.40 \%,-8.24$ to $5.65 \%$, in $\mathrm{E}_{1}, \mathrm{E}_{2}, \mathrm{E}_{3}, \mathrm{E}_{4}$, respectively. In pooled minimum significant and negative heterobeltiosis $\left(\mathrm{H}_{1}\right)$ was exhibited by the cross AT-322 x RT-54, AT341 x G.Til-1, AT-306 x AT-285, AT-253 x RT-54 and AT-306 x RT-54. Maximum heterobeltiosis was observed in the cross IS209 x G.Til-10. The cross combination AT$306 \times$ AT-285 recorded the highest desirable standard heterosis $\left(\mathrm{H}_{2} \mathrm{C}_{1}\right)$ over G.Til-2 followed by AT-322 x RT-54, AT-341 x AT285, Bhuva-2 x AT-285 and AT-341 x G. Til1 in across the environments. In case of the cross combination AT-306 $\times$ AT-285 recorded the highest desirable standard heterosis $\left(\mathrm{H}_{2} \mathrm{C}_{2}\right)$ over G.Til-3 followed by AT-322 x RT-54, AT-341 x AT-285, Bhuva-2 x AT-285 and AT-265 x AT-285 in across the environments. Out 40 of $\mathrm{F}_{1} \mathrm{~s}$, significant and negative standard heterosis $\left(\mathrm{H}_{2} \mathrm{C}_{1}\right)$ crosses was recorded in 20, 14, 0, 35 and 4 in $\mathrm{E}_{1}, \mathrm{E}_{2}, \mathrm{E}_{3}, \mathrm{E}_{4}$ and across the environments, respectively. Likewise significant and negative standard heterosis $\left(\mathrm{H}_{2} \mathrm{C}_{2}\right)$ crosses was recorded in 9, 5, 11,25 and 1 in $E_{1}, E_{2}, E_{3}, E_{4}$ and across the environments, respectively.

Positive heterosis for plant height (Table 4) is desirable for developing hybrid with tall plant type with high seed yield per plant. The tall parent was considered as better parent to estimate the heterobeltiosis for plant height. The heterobeltiosis ranged from IS-209 $\mathrm{x}$ G.Til-10 in $E_{1}$, Bhuva-2 $x$ G.Til-10 in $E_{2}$, Bhuva-2 x G.Til-10 in $\mathrm{E}_{3}$, Bhuva-2 x G.Til-10 in $\mathrm{E}_{4}$, and IS-209 x G.Til-10 and Bhuva-2 $\mathrm{x}$ G.Til-10 across the environments. Out of 40 crosses, significant and positive heterobeltiosis crosses was recorded in 20,39, 20,37 and 16 in $E_{1}, E_{2}, E_{3}, E_{4}$ and across the environments, respectively. Out of 40 significant cross, combinations the magnitude of standard heterosis across the environments over $\mathrm{H}_{2} \mathrm{C}_{1}$ and $\mathrm{H}_{2} \mathrm{C}_{2}$ ranged from -24.34 (AT$253 \times$ RT-54) to $14.28 \%$ (IS-209 x G.Til-10) and -20.53 (AT-253 x RT-54) to $20.03 \%$ (IS209 x G.Til-10), respectively. 
Table.1 Analysis of variance (mean squares) for days to flowering and days to maturity under individual environments and pooled over environments

\begin{tabular}{|c|c|c|c|c|c|c|c|c|c|c|c|c|c|c|c|c|}
\hline \multirow{2}{*}{ Source } & \multirow[t]{2}{*}{ d.f. } & \multicolumn{5}{|c|}{ Days to flowering } & \multicolumn{5}{|c|}{ Days to maturity } & \multicolumn{5}{|c|}{ Seed yield per plant (g) } \\
\hline & & E. & E. & E. & & Pooled & E. & E. & $\mathbf{E}$ & $\mathbf{E}$ & Pooled & E. & E. & E. & E. & Ponled \\
\hline Environment & 3 & - & - & - & - & $203.82 * *$ & - & - & - & - & $705.72 * *$ & - & - & - & - & $82.83 * *$ \\
\hline Replications & 2 & $21.91 *$ & $22.67 * *$ & $8.01 *$ & $17.67 * *$ & $65.35 * *$ & $24.13 * *$ & $31.60 * *$ & $64.64 * *$ & $127.60 * *$ & $201.53 * *$ & $1.71 * *$ & $3.27 * *$ & $1.59 * *$ & $2.52 * *$ & $6.98^{* * *}$ \\
\hline Replications & 6 & - & - & - & - & 1.63 & - & - & - & - & $15.47 * *$ & - & - & - & & 0.007 \\
\hline Genotypes & 53 & $8.45 * *$ & $11.54 * *$ & $11.21 * *$ & $9.61 * *$ & $10.15^{* *}$ & $22.74 * *$ & $16.51 * *$ & $13.81 * *$ & $19.39 * *$ & $16.42 * *$ & $11.19 * *$ & $26.39 * *$ & $2.97 * *$ & $4.98 * *$ & $9.47^{* * * 6}$ \\
\hline Parents & 13 & $12.52 *$ & $21.36 * *$ & $8.76^{* *}$ & $13.62 * *$ & $15.20 * *$ & $31.99 * *$ & $13.89 * *$ & $13.01 * *$ & $7.64 * *$ & $11.47 * *$ & $6.65 * *$ & $18.76^{* *}$ & $2.28 * *$ & $2.51 * *$ & $6.62^{* * * *}$ \\
\hline Females (F) & 9 & $14.01 *$ & $12.16 * *$ & $7.78 * *$ & $10.83 * *$ & $12.28 * *$ & $34.68 * *$ & $7.29 *$ & $15.04 * *$ & $7.54 * *$ & $9.36 * *$ & $6.25 * *$ & $8.16 * *$ & $3.00 * *$ & $3.21 * *$ & $5.74 * * *$ \\
\hline Males (M) & 3 & $12.08 *$ & $53.00 * *$ & $12.11 * *$ & $14.53 * *$ & $24.76^{* *}$ & $33.64 * *$ & $38.08 * *$ & $11.22 *$ & $10.33 * *$ & $21.04 * *$ & $7.90^{*}$ & $30.74 * *$ & 0.36 & $1.14 * *$ & $7.42 *$ \\
\hline Hybrids & 39 & $5.39 * *$ & $8.00 * *$ & $10.67 * *$ & $7.52 * *$ & $7.57 * *$ & $19.96 * *$ & $15.48 * *$ & $12.12 * *$ & $20.74 * *$ & $16.16^{* *}$ & $12.87 * *$ & $29.27 * *$ & $3.23 * *$ & $5.77 * *$ & $10.60 \% * * *$ \\
\hline P vs H & 1 & $75.09 *$ & $22.23 * *$ & $63.91 * *$ & $38.96^{* *}$ & $63.56^{* *}$ & $10.80 * *$ & $90.54 * *$ & $90.16 * *$ & $119.31 * *$ & $91.97 * *$ & $4.75^{* *}$ & $13.23 *$ & $2.06^{* *}$ & $6.12 * *$ & 2.40 \\
\hline$P \times E$ & 39 & - & - & - & - & $3.54 * *$ & - & - & - & - & $21.11 * *$ & - & - & - & - & $3.44 * * *$ \\
\hline $\mathrm{H} \times \mathrm{E}$ & 117 & - & - & - & - & $2.95 * *$ & - & - & - & - & $39.70 * *$ & - & - & - & - & $6.44^{* * *}$ \\
\hline$(\mathbf{P}$ vs H) $\times \mathrm{E}$ & 3 & - & - & - & - & $3.17 * *$ & - & - & - & - & $197.12 * *$ & - & - & - & - & $6.32 * * *$ \\
\hline
\end{tabular}

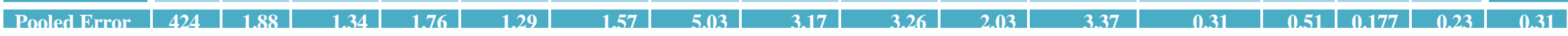

\begin{tabular}{|c|c|c|c|c|c|c|c|c|c|c|c|}
\hline \multirow{2}{*}{ Source } & \multirow[t]{2}{*}{ d.f. } & \multicolumn{5}{|c|}{ Plant height (cm) } & \multicolumn{5}{|c|}{ Height to first capsule } \\
\hline & & $\mathbf{E}_{1}$ & $\mathbf{E}_{2}$ & $\mathbf{E}_{3}$ & $\mathbf{E}_{4}$ & Pooled & $\mathbf{E}_{1}$ & $\mathbf{E}_{2}$ & $\mathbf{E}_{3}$ & $\mathbf{E}_{4}$ & Pooled \\
\hline Environments (E) & 3 & - & - & - & - & $1814.92 * *$ & - & - & - & - & $1607.89 * * *$ \\
\hline Replications & 2 & $46.69 * *$ & $29.66^{*}$ & $51.51 * *$ & $52.61 * *$ & $162.11 * *$ & $10.96^{*}$ & 15.55 & $35.18 * *$ & $17.21^{*}$ & $64.20 * *$ \\
\hline Replications *E & 6 & - & - & - & - & $6.08 * *$ & - & - & - & - & $4.89 * * *$ \\
\hline Genotypes & 53 & $307.69 * *$ & $162.18 * *$ & $234.94 * *$ & $181.90 * *$ & $229.80 * *$ & $143.37 * *$ & $121.21 * *$ & $98.33 * *$ & $125.15^{* *}$ & $124.57 * *$ \\
\hline Parents & 13 & $340.71 * *$ & $258.31 * *$ & $246.52 * *$ & $229.42 * *$ & $326.37 * *$ & $230.05 * *$ & $236.38 * *$ & $162.57 * *$ & $210.17 * *$ & $258.61 * *$ \\
\hline Females (F) & 9 & $376.96 * *$ & $258.12 * *$ & $262.24 * *$ & $206.54 * *$ & $329.12 * *$ & $258.34 * *$ & $244.40 * *$ & $180.41 * *$ & $210.98 * *$ & $270.19 * *$ \\
\hline Males (M) & 3 & $264.44 * *$ & $267.34 * *$ & $223.06^{* * *}$ & $195.93 * *$ & $301.24 * *$ & $177.69 * *$ & $219.64 * *$ & $146.04 * *$ & $189.82 * *$ & $231.72 * *$ \\
\hline Hybrids & 39 & $299.25 * *$ & $132.74 * *$ & $235.23 * *$ & $167.64 * *$ & $201.56 * *$ & $110.88 * *$ & $85.62 * *$ & $69.53 * *$ & $98.35 * *$ & $81.75^{* * *}$ \\
\hline $\mathbf{P}$ vs $\mathrm{H}$ & 1 & $207.34 * *$ & $60.88 * *$ & $72.94 * *$ & $120.35 * *$ & 1.45 & $283.75 * *$ & 12.13 & $386.34 * *$ & $64.93 * *$ & $51.92 *$ \\
\hline $\mathbf{P x E}$ & 39 & - & - & - & - & $65.77 * *$ & - & - & - & - & $21.11 * *$ \\
\hline $\mathrm{H} \times \mathrm{E}$ & 117 & - & - & - & - & $74.82 * *$ & - & - & - & - & $39.70 * *$ \\
\hline$(\mathrm{P}$ vs H) x E & 3 & - & - & - & - & $152.38 * *$ & - & - & - & - & $197.12 * *$ \\
\hline Pooled Error & 424 & 5.23 & 8.38 & 5.42 & 9.75 & 7.16 & 2.80 & 5.81 & 2.38 & 5.21 & 4.05 \\
\hline
\end{tabular}

*, ** Significant against pooled error at $5 \%$ and $1 \%$, respectively. 
Table.2 Heterobeltiosis and standard heterosis over G.Til-2 and G.Til-3 under individual environments and pooled over environments for days to flowering

\begin{tabular}{|c|c|c|c|c|c|c|c|c|c|c|c|c|c|c|c|c|}
\hline \multirow{2}{*}{$\begin{array}{l}\text { Sr. } \\
\text { No. }\end{array}$} & \multirow[t]{2}{*}{ Crosses } & \multicolumn{5}{|c|}{ Heterobeltiosis $\left(\mathbf{H}_{1}\right)$} & \multicolumn{5}{|c|}{ Std. Heterosis over G.Til-2 $\left(\mathrm{H}_{2} \mathrm{C}_{1}\right)$} & \multicolumn{5}{|c|}{ Std. Heterosis over G.Til-3 $\left(\mathrm{H}_{2} \mathrm{C}_{2}\right)$} \\
\hline & & $\mathbf{E}_{1}$ & $\mathbf{E}_{2}$ & $\mathbf{E}_{3}$ & $\mathbf{E}_{4}$ & Pooled & $\mathbf{E}_{1}$ & $\mathbf{E}_{2}$ & $\mathbf{E}_{3}$ & $\mathbf{E}_{4}$ & Pooled & $\mathbf{E}_{1}$ & $\mathbf{E}_{2}$ & $\mathbf{E}_{3}$ & $\mathbf{E}_{4}$ & Pooled \\
\hline 1 & AT-253 $\times$ AT- 285 & 0.86 & $6.03 * *$ & $-6.90^{* *}$ & -1.72 & -1.28 & -2.50 & 0.00 & 2.86 & -1.72 & -0.43 & 1.74 & 2.50 & -3.57 & 0.88 & 0.43 \\
\hline 2 & AT-253 x G.Til-1 & 0.00 & $7.69^{* * *}$ & $-9.40 * *$ & 0.00 & 3.10 & -2.50 & 2.44 & 0.95 & 0.86 & 0.43 & 1.74 & $5.0^{* * *}$ & $-5.36 * *$ & $3.54 *$ & 1.30 \\
\hline 3 & AT-253 x G.Til-10 & -3.31 & 0.83 & $-4.13^{*}$ & $-3.31 *$ & -0.21 & -2.50 & -0.81 & $10.48^{* * *}$ & 0.86 & 1.72 & 1.74 & 1.67 & 3.57 & $3.54 *$ & 2.61 \\
\hline 4 & AT-253 x RT-54 & $-4.96 * *$ & -0.83 & $-15.70^{* *}$ & $-6.61 * *$ & -4.86 & $-4.17^{*}$ & -2.44 & -2.86 & -2.59 & -3.02 & 0.00 & 0.00 & $-8.93 * *$ & 0.00 & -2.17 \\
\hline 5 & AT-265 x AT-285 & -0.86 & $3.45^{*}$ & $-11.21 * *$ & $-4.31 *$ & -4.06 & $-4.17^{*}$ & -2.44 & -1.90 & $-4.31^{*}$ & -3.23 & 0.00 & 0.00 & $-8.04 * *$ & -1.77 & -2.39 \\
\hline 6 & AT-265 x G.Til-1 & $-4.27 *$ & -2.56 & $-11.11^{* * *}$ & $-5.98 * *$ & -2.65 & $-6.67 * *$ & $-7.32 * *$ & -0.95 & $-5.17^{* * *}$ & -5.17 & -2.61 & $-5.00 * *$ & $-7.14 * *$ & -2.65 & -4.35 \\
\hline 7 & AT-265 x G.Til-10 & -1.68 & $4.20^{*}$ & $-8.40^{* * *}$ & $-5.88 * *$ & -1.49 & -2.50 & 0.81 & 3.81 & $-3.45^{*}$ & -0.43 & 1.74 & $3.33^{*}$ & -2.68 & -0.88 & 0.43 \\
\hline 8 & AT-265 x RT-54 & $-4.20^{*}$ & -1.68 & $-13.45^{* *}$ & $-4.20^{*}$ & -4.48 & $-5.00^{* *}$ & $-4.88 * *$ & -1.90 & -1.72 & -3.45 & -0.87 & -2.50 & $-8.04 * *$ & 0.88 & -2.61 \\
\hline 9 & AT-306 x AT-285 & -3.45 & 1.72 & $-12.07 * *$ & 0.00 & -4.27 & $-6.67^{* *}$ & $-4.07^{*}$ & -2.86 & 0.00 & -3.45 & -2.61 & -1.67 & $-8.93 * *$ & 2.65 & -2.61 \\
\hline 10 & AT-306 x G.Til-1 & -3.42 & $-5.13^{* * *}$ & -11.11 ** & $-6.84 * *$ & -3.32 & $-5.83 * *$ & $-9.76^{* *}$ & -0.95 & $-6.03 * *$ & $-5.82^{*}$ & -1.74 & $-5.00 * *$ & $-7.14 * *$ & $-3.54 *$ & -5.00 \\
\hline 11 & AT-306 x G.Til- 10 & -3.10 & 0.00 & $-11.63 * *$ & -2.33 & -2.37 & $4.17^{*}$ & $4.88^{* *}$ & $8.57 * *$ & $8.62^{* * *}$ & $6.47 *$ & $8.70^{* * *}$ & $10.00^{* * *}$ & 1.79 & $11.50^{* * *}$ & $7.39^{* *}$ \\
\hline 12 & AT-306 x RT-54 & $-6.50 * *$ & $-4.07 *$ & $-15.45^{* * *}$ & $-7.32^{* * *}$ & $-9.26^{* * *}$ & $-4.17^{*}$ & $-4.07 *$ & -0.95 & -1.72 & -2.80 & 0.00 & -1.67 & $-7.14 * *$ & 0.88 & -1.96 \\
\hline 13 & AT-307 x AT-285 & $-4.31 *$ & $6.03 * *$ & $-14.66^{* * *}$ & 0.86 & -3.85 & $-7.50^{* * *}$ & 0.00 & $-5.71 * *$ & 0.86 & -3.02 & -3.48 & 2.50 & $-11.61^{* * *}$ & $3.54 *$ & -2.17 \\
\hline 14 & AT-307 x G.Til-1 & 0.00 & $5.13 * *$ & $-14.53^{* *}$ & 1.71 & 1.55 & -2.50 & 0.00 & $-4.76^{*}$ & 2.59 & -1.08 & 1.74 & 2.50 & $-10.71 * *$ & $5.31^{* *}$ & -0.22 \\
\hline 15 & AT-307 x G.Til-10 & $-4.72 * *$ & $-7.87 * *$ & $-8.66 * *$ & $-10.24 * *$ & -4.88 & 0.83 & $-4.88 * *$ & $10.48 * *$ & -1.72 & 0.86 & $5.22 * *$ & -2.50 & 3.57 & 0.88 & 1.74 \\
\hline 16 & AT-307 x RT-54 & $-5.69 * *$ & $3.25^{*}$ & $-17.07 * *$ & $-5.69 * *$ & $-6.30^{*}$ & -3.33 & $3.25 *$ & -2.86 & 0.00 & -0.65 & 0.87 & $5.83^{* * *}$ & $-8.93 * *$ & 2.65 & 0.22 \\
\hline 17 & AT-319 x AT-285 & -0.87 & $7.83^{* *}$ & $-10.43^{* *}$ & $3.48 *$ & 2.22 & $-5.0^{* * *}$ & 0.81 & -1.90 & 2.59 & -0.86 & -0.87 & $3.33^{*}$ & $-8.04 * *$ & $5.31^{* * *}$ & 0.00 \\
\hline 18 & AT-319 x G.Til-1 & -3.48 & -0.87 & $-9.57 * *$ & -0.87 & -1.56 & $-7.50 * *$ & $-7.32 * *$ & -0.95 & -1.72 & -4.53 & -3.48 & $-5.00^{* * *}$ & $-7.14 * *$ & 0.88 & -3.70 \\
\hline 19 & AT- 319 x G.Til- 10 & $4.35^{*}$ & $6.96^{* * *}$ & -2.61 & $4.35^{*}$ & $5.56^{*}$ & 0.00 & 0.00 & $6.67 * *$ & $3.45^{*}$ & 2.37 & $4.35^{*}$ & 2.50 & 0.00 & $6.19^{* * *}$ & 3.26 \\
\hline 20 & AT-319 x RT-54 & -2.61 & $4.35^{*}$ & $-8.70 * *$ & -1.74 & 0.00 & $-6.67 * *$ & -2.44 & 0.00 & -2.59 & -3.02 & -2.61 & 0.00 & $-6.25 * *$ & 0.00 & -2.17 \\
\hline 21 & AT-322 $\times$ AT- 285 & -1.72 & $3.45^{*}$ & $-8.62^{* * *}$ & $-6.03 * *$ & -4.06 & $-5.0^{* * *}$ & -2.44 & 0.95 & $-6.03 * *$ & -3.23 & -0.87 & 0.00 & $-5.36 * *$ & $-3.54 *$ & -2.39 \\
\hline 22 & AT-322 x G.Til-1 & -1.71 & $7.69 * *$ & $-5.98 * *$ & 2.56 & 4.20 & $-4.17^{*}$ & 2.44 & $4.76^{*}$ & $3.45^{*}$ & 1.51 & 0.00 & $5.00^{* * *}$ & -1.79 & $6.19^{* * *}$ & 2.39 \\
\hline 23 & AT- $322 \times$ G.Til- 10 & -0.78 & 0.00 & $-14.73 * *$ & $-6.98 * *$ & -2.21 & $6.67 * *$ & $4.88^{* *}$ & $4.76^{*}$ & $3.45^{*}$ & 4.96 & $11.30^{* *}$ & $7.50^{* * *}$ & -1.79 & $6.19^{* * *}$ & $5.87 *$ \\
\hline 24 & AT-322 x RT-54 & -3.25 & $-4.88 * *$ & $-14.63 * *$ & $-9.76 * *$ & $-9.05 * *$ & -0.83 & $-4.88^{* *}$ & 0.00 & $-4.31^{*}$ & -2.59 & 3.48 & -2.50 & $-6.25 * *$ & -1.77 & -1.74 \\
\hline 25 & AT-341 x AT-285 & $-4.31 *$ & $6.03^{* * *}$ & -3.45 & -0.86 & 0.22 & $-7.50^{* *}$ & 0.00 & $6.67 * *$ & -0.86 & -0.65 & -3.48 & 2.50 & 0.00 & 1.77 & 0.22 \\
\hline 26 & AT-341 x G.Til-1 & -0.85 & 0.00 & $-11.11^{* *}$ & $-7.69 * *$ & -1.55 & -3.33 & $-4.88 * *$ & -0.95 & $-6.90 * *$ & -4.09 & 0.87 & -2.50 & $-7.14 * *$ & $-4.42 *$ & -3.26 \\
\hline 27 & AT-341 x G.Til-10 & 0.85 & 2.54 & -3.39 & 1.69 & 3.04 & -0.83 & -1.63 & $8.57 * *$ & $3.45^{*}$ & 2.16 & 3.48 & 0.83 & 1.79 & $6.19^{* *}$ & 3.04 \\
\hline 28 & AT-341 x RT-54 & -2.54 & -0.85 & $-9.32 * *$ & $-7.63 * *$ & -2.61 & $-4.17^{*}$ & $-4.88 * *$ & 1.90 & $-6.03 * *$ & -3.45 & 0.00 & -2.50 & $-4.46^{*}$ & $-3.54 *$ & -2.61 \\
\hline 29 & Bhuva-2 $\mathrm{x}$ AT-285 & -3.45 & $3.45^{*}$ & $-12.93 * *$ & $-6.90 * *$ & -2.65 & $-6.67 * *$ & -2.44 & -3.81 & $-6.90 * *$ & -4.96 & -2.61 & 0.00 & $-9.82 * *$ & $-4.42^{*}$ & -4.13 \\
\hline 30 & Bhuva-2 x G.Til-1 & 0.00 & $7.76^{* *}$ & -3.45 & 2.59 & 4.42 & -3.33 & 1.63 & $6.67 * *$ & 2.59 & 1.72 & 0.87 & $4.17^{*}$ & 0.00 & $5.31^{* *}$ & 2.61 \\
\hline 31 & Bhuva-2 $\times$ G.Til-10 & $4.31^{*}$ & $12.07 * *$ & $5.17 * *$ & $5.17 * *$ & $9.27 * *$ & 0.83 & $5.69 * *$ & $16.19 * *$ & $5.17 * *$ & $6.68^{*}$ & $5.22 * *$ & $8.33^{* * *}$ & $8.93 * *$ & $7.96 * *$ & $7.61^{* * *}$ \\
\hline 32 & Bhuva-2 x RT-54 & -0.86 & $3.45^{*}$ & $-12.07 * *$ & $-4.31 *$ & -1.10 & $-4.17^{*}$ & -2.44 & -2.86 & $-4.31^{*}$ & -3.45 & 0.00 & 0.00 & $-8.93 * *$ & -1.77 & -2.61 \\
\hline 33 & Khadkala-S x AT285 & -2.65 & 2.65 & $-9.73 * *$ & $-6.19 * *$ & -2.47 & $-8.33 * *$ & $-5.69 * *$ & -2.86 & $-8.62^{* * *}$ & $-6.47^{*}$ & $-4.35^{*}$ & $-3.33^{*}$ & $-8.93 * *$ & $-6.19 * *$ & $-5.65^{*}$ \\
\hline 34 & Khadkala-S x G.Till & 2.65 & $7.96 * *$ & $-8.85 * *$ & 2.65 & 2.70 & -3.33 & -0.81 & -1.90 & 0.00 & -1.51 & 0.87 & 1.67 & $-8.04 * *$ & 2.65 & -0.65 \\
\hline 35 & Khadkala-S x GTil10 & $8.85^{* * *}$ & $16.81 * *$ & 3.54 & $9.73 * *$ & $11.46 * *$ & 2.50 & $7.32^{* * *}$ & $11.43 * *$ & $6.90^{* * *}$ & $6.90 * *$ & $6.96^{* * *}$ & $10.00 * *$ & $4.46^{*}$ & $9.73^{* *}$ & $7.83^{* * *}$ \\
\hline 36 & Khadkala-S x RT-54 & $4.42^{*}$ & $7.96 * *$ & 0.00 & 0.88 & 4.94 & -1.67 & -0.81 & $7.62 * *$ & -1.72 & 0.65 & 2.61 & 1.67 & 0.89 & 0.88 & 1.52 \\
\hline 37 & IS-209 x AT-285 & 0.86 & $6.90^{* * *}$ & $-10.34 * *$ & $-3.45^{*}$ & -2.14 & -2.50 & 0.81 & -0.95 & $-3.45 *$ & -1.51 & 1.74 & $3.33^{*}$ & $-7.14 * *$ & -0.88 & -0.65 \\
\hline 38 & IS-209 x G.Til-1 & $-4.31 *$ & $3.45^{*}$ & $-12.07 * *$ & $-3.45^{*}$ & -1.55 & -7.50 ** & -2.44 & -2.86 & $-3.45^{*}$ & -4.09 & -3.48 & 0.00 & $-8.93 * *$ & -0.88 & -3.26 \\
\hline 39 & IS-209 x G.Til-10 & $5.17 * *$ & $14.66^{* * *}$ & 0.86 & $6.03 * *$ & $6.00^{*}$ & 1.67 & $8.13^{* * *}$ & $11.43 * *$ & $6.03 * *$ & $6.68 *$ & $6.09 * *$ & $10.00^{* * *}$ & $4.46^{*}$ & $8.85^{* * *}$ & $7.61^{* * *}$ \\
\hline 40 & IS-209 x RT-54 & 0.00 & $6.03 * *$ & $-6.03 * *$ & 0.00 & -0.64 & -3.33 & 0.00 & 3.81 & 0.00 & 0.00 & 0.87 & 2.50 & -2.68 & 2.65 & 0.87 \\
\hline & Min. & -6.50 & -7.87 & -17.07 & -10.24 & -9.26 & -8.33 & -9.76 & -5.71 & -8.62 & -6.47 & -4.35 & -7.50 & -11.61 & -6.19 & -5.65 \\
\hline & Max. & 8.85 & 16.81 & 5.17 & 9.73 & 11.46 & 6.67 & 8.13 & 16.19 & 8.62 & 6.90 & 11.30 & 10.83 & 8.93 & 11.50 & 7.83 \\
\hline & Mean heterosis & -1.29 & 3.54 & -8.85 & -2.21 & -0.65 & -3.23 & -1.02 & 2.10 & -0.86 & -0.85 & 0.98 & 1.46 & -4.29 & 1.77 & 0.02 \\
\hline Desi & ble heterosis & 9 & 4 & 32 & 20 & 3 & 19 & 11 & 2 & 13 & 2 & 1 & 4 & 24 & 6 & 1 \\
\hline
\end{tabular}


Table.3 Heterobeltiosis and standard heterosis over G.Til-2 and G.Til-3 under individual environments and pooled over environments for days to maturity

\begin{tabular}{|c|c|c|c|c|c|c|c|c|c|c|c|c|c|c|c|c|}
\hline \multirow{2}{*}{$\begin{array}{l}\text { Sr. } \\
\text { No. }\end{array}$} & \multirow[t]{2}{*}{ Crosses } & \multicolumn{5}{|c|}{ Heterobeltiosis $\left(\mathbf{H}_{\mathbf{1}}\right)$} & \multicolumn{5}{|c|}{ Std. Heterosis over G.Til-2 $\left(\mathrm{H}_{2} \mathrm{C}_{1}\right)$} & \multicolumn{5}{|c|}{ Std. Heterosis over G.Til-3 $\left(\mathrm{H}_{2} \mathrm{C}_{2}\right)$} \\
\hline & & $E_{1}$ & $\mathbf{E}_{2}$ & $\mathbf{E}_{3}$ & $\mathbf{E}_{4}$ & Pooled & $\mathbf{E}_{1}$ & $\mathbf{E}_{2}$ & $\mathbf{E}_{3}$ & $\mathbf{E}_{4}$ & Pooled & $\mathbf{E}_{1}$ & $\mathbf{E}_{2}$ & $\mathbf{E}_{3}$ & $\mathbf{E}_{4}$ & Pooled \\
\hline 1 & AT-253 $\times$ AT- 285 & 1.57 & $5.12 * *$ & $-5.91 * *$ & $-1.97 *$ & 2.01 & -1.53 & $2.30^{*}$ & 3.46 ** & $-3.86 * *$ & 0.00 & 0.00 & $3.49 * *$ & 1.27 & -1.58 & 0.80 \\
\hline 2 & AT- $253 \times$ G.Til-1 & 0.39 & $1.97 *$ & $-4.72 * *$ & -1.57 & -0.89 & $-2.67 *$ & -0.77 & 4.76 ** & $-3.47 * *$ & -0.69 & -1.16 & 0.39 & $2.54^{*}$ & -1.19 & 0.10 \\
\hline 3 & AT- $253 \times$ G.Til- 10 & $2.36^{*}$ & $2.76 * *$ & $-3.15 * *$ & -1.18 & 0.20 & -0.76 & 0.00 & 6.49 ** & $-3.09 * *$ & 0.49 & 0.78 & 1.16 & $4.24 * *$ & -0.79 & 1.29 \\
\hline 4 & AT- $253 \times$ RT-54 & 0.79 & $3.17 * *$ & -6.75 ** & -5.56 ** & -2.85 & $-3.05 * *$ & -0.38 & 1.73 & $-8.11 * *$ & -2.57 & -1.55 & 0.78 & -0.42 & $-5.93 * *$ & -1.79 \\
\hline 5 & AT-265 x AT-285 & 0.40 & $1.99^{*}$ & $-7.57^{* * *}$ & $-4.38 * *$ & -1.31 & $-3.82^{* * *}$ & $-1.92^{*}$ & 0.43 & $-7.34 * *$ & -3.26 & $-2.33^{*}$ & -0.78 & -1.69 & $-5.14 * *$ & -2.49 \\
\hline 6 & AT-265 x G.Til-1 & 0.80 & 1.59 & $-5.98 * *$ & $-4.38 * *$ & -2.57 & $-3.44 * *$ & $-2.30^{*}$ & $2.16^{*}$ & $-7.34 * *$ & -2.86 & -1.94 & -1.16 & 0.00 & $-5.14 * *$ & -2.09 \\
\hline 7 & AT- $265 \times$ x G.Til- 10 & $2.79^{*}$ & $4.78^{* *}$ & $-4.38 * *$ & -3.19 ** & -0.59 & -1.53 & 0.77 & $3.9^{* *}$ & $-6.18 * *$ & -0.89 & 0.00 & $1.94 *$ & 1.69 & $-3.95 * *$ & -0.10 \\
\hline 8 & AT- $265 \times$ RT- 54 & 0.80 & $2.79 * *$ & $-5.98 * *$ & $-5.18 * *$ & -2.48 & $-3.44 * *$ & -1.15 & $2.16^{*}$ & $-8.11 * *$ & -2.76 & -1.94 & 0.00 & 0.00 & $-5.93 * *$ & -1.99 \\
\hline 9 & AT-306 $\times$ AT- 285 & -1.57 & $-1.96^{*}$ & $-10.20 * *$ & $-8.24 * *$ & -2.92 & $-4.20 * *$ & $-4.21 * *$ & -0.87 & $-9.65 * *$ & $-4.84 * *$ & $-2.71 *$ & $-3.10 * *$ & $-2.97 * *$ & $-7.51 * *$ & $-4.08^{*}$ \\
\hline 10 & AT-306 x G.Til-1 & $-2.26^{*}$ & $-2.64 * *$ & $-9.06 * *$ & $-5.66 * *$ & -0.69 & -1.15 & -1.15 & $4.33 * *$ & $-3.47 * *$ & -0.49 & 0.39 & 0.00 & $2.12^{*}$ & -1.19 & 0.30 \\
\hline 11 & AT- $306 \times$ G.Til- 10 & 0.37 & 1.11 & $-10.74 * *$ & $-2.59 * *$ & 2.34 & $3.44 * *$ & $4.60 * *$ & $4.33 * *$ & 1.54 & 3.46 & $5.04 * *$ & $5.81 * *$ & $2.12^{*}$ & $3.95 * *$ & $4.28^{*}$ \\
\hline 12 & AT- 306 x RT-54 & 1.19 & 1.59 & $-8.33 * *$ & $-1.98^{*}$ & -2.85 & $-2.67 *$ & $-1.92^{*}$ & 0.00 & $-4.63 * *$ & -2.37 & -1.16 & -0.78 & $-2.12^{*}$ & $-2.37 * *$ & -1.59 \\
\hline 13 & AT- 307 x AT-285 & -1.57 & $4.31 * *$ & $-8.63 * *$ & $-3.14 * *$ & 0.40 & $-4.20 * *$ & $1.92^{*}$ & 0.87 & $-4.63 * *$ & -1.58 & $-2.71^{*}$ & $3.10 * *$ & -1.27 & $-2.37 * *$ & -0.80 \\
\hline 14 & AT-307 x G.Til-1 & $-3.40 * *$ & $-5.28 * *$ & $-13.21 * *$ & $-6.42 * *$ & -2.18 & $-2.29 *$ & $-3.83 * *$ & -0.43 & $-4.25 * *$ & -2.76 & -0.78 & $-2.71 * *$ & $-2.54 *$ & $-1.98 *$ & -1.99 \\
\hline 15 & AT-307 x G.Til-10 & 1.89 & 0.38 & $-9.43 * *$ & $-4.53 * *$ & 2.18 & $3.05 * *$ & $1.92 *$ & $3.90 * *$ & $-2.32 * *$ & 1.58 & $4.65 * *$ & $3.1 * *$ & 1.69 & 0.00 & 2.39 \\
\hline 16 & AT-307 x RT-54 & $2.38 *$ & 1.59 & $-7.94 * *$ & -1.59 & -1.29 & -1.53 & $-1.92^{*}$ & 0.43 & $-4.25 * *$ & -1.88 & 0.00 & -0.78 & -1.69 & $-1.98^{*}$ & -1.09 \\
\hline 17 & AT- $319 \times$ AT- 285 & -0.78 & 0.00 & $-9.80 * *$ & -0.78 & -0.20 & $-3.44 * *$ & $-2.30^{*}$ & -0.43 & $-2.32 * *$ & -2.17 & -1.94 & -1.16 & $-2.54 *$ & 0.00 & -1.39 \\
\hline 18 & AT-319 x G.Til-1 & $-4.91 * *$ & $-2.64 * *$ & $-11.32 * *$ & $-7.92 * *$ & -2.56 & $-3.82 * *$ & -1.15 & 1.73 & $-5.79 * *$ & -2.37 & $-2.33^{*}$ & 0.00 & -0.42 & $-3.56 * *$ & -1.59 \\
\hline 19 & AT-319 x G.Til-10 & -1.46 & $-2.55 * *$ & $-12.77 * *$ & -7.30 ** & -2.37 & $3.05 * *$ & $2.30^{*}$ & $3.46 * *$ & $-1.93^{*}$ & 1.68 & $4.65 * *$ & $3.49 * *$ & 1.27 & 0.40 & 2.49 \\
\hline 20 & AT-319 x RT-54 & 1.19 & 0.00 & $-7.54 * *$ & 0.00 & -2.55 & $-2.67 *$ & $-3.45 * *$ & 0.87 & $-2.70 * *$ & -2.07 & -1.16 & $-2.33^{*}$ & -1.27 & -0.40 & -1.29 \\
\hline 21 & AT- $322 \times$ AT- 285 & -1.57 & 1.57 & $-7.84 * *$ & $-6.27 * *$ & -0.91 & $-4.20 * *$ & -0.77 & 1.73 & $-7.72 * *$ & -2.86 & $-2.71^{*}$ & 0.39 & -0.42 & $-5.53 * *$ & -2.09 \\
\hline 22 & AT- $322 \times$ G.Til-1 & $-2.64 *$ & $-4.15 * *$ & -8.30 ** & $-7.92 * *$ & -1.58 & -1.53 & $-2.68 * *$ & $5.19^{* *}$ & $-5.79 * *$ & -1.38 & 0.00 & -1.55 & $2.97 * *$ & $-3.56 * *$ & -0.60 \\
\hline 23 & AT- $322 \times$ G.Til- 10 & $2.96 * *$ & -1.48 & $-11.11 * *$ & $-8.15 * *$ & -1.43 & $6.11 * *$ & $1.92^{*}$ & $3.90 * *$ & $-4.25 * *$ & 1.88 & $7.75^{* * *}$ & $3.10 * *$ & 1.69 & $-1.98^{*}$ & 2.69 \\
\hline 24 & AT-322 x RT-54 & -0.79 & 1.59 & $-9.52 * *$ & $-5.95 * *$ & $-4.62 * *$ & $-4.58 * *$ & $-1.92^{*}$ & -1.30 & $-8.49 * *$ & $-4.15^{*}$ & $-3.10 * *$ & -0.78 & $-3.39 * *$ & $-6.32 * *$ & -3.38 \\
\hline 25 & AT- $341 \times$ AT- 285 & 0.00 & 1.62 & $-3.24 * *$ & $-4.86 * *$ & -2.11 & $-5.73 * *$ & $-3.83 * *$ & $3.46 * *$ & $-9.27 * *$ & $-4.05^{*}$ & $-4.26 * *$ & $-2.71 * *$ & 1.27 & $-7.11 * *$ & -3.28 \\
\hline 26 & AT-341 x G.Til-1 & 4.86 ** & $2.43^{*}$ & $-6.88 * *$ & $-4.05 * *$ & -2.97 & -1.15 & $-3.07 * *$ & -0.43 & $-8.49 * *$ & -3.36 & 0.39 & $-1.94 *$ & $-2.54 *$ & $-6.32 * *$ & -2.59 \\
\hline 27 & AT-341 x G.Til-10 & 4.86 ** & $6.07 * *$ & -0.40 & 0.40 & 0.59 & -1.15 & 0.38 & $6.49 * *$ & $-4.25 * *$ & 0.20 & 0.39 & 1.55 & $4.24 * *$ & $-1.98 *$ & 1.00 \\
\hline 28 & AT-341 x RT-54 & $5.26^{* * *}$ & $3.24 * *$ & $-4.45 * *$ & $-3.64 * *$ & -1.98 & -0.76 & $-2.30^{*}$ & $2.16^{*}$ & $-8.11 * *$ & -2.37 & 0.78 & -1.16 & 0.0 & $-5.93 * *$ & -1.59 \\
\hline 29 & Bhuva-2 x AT-285 & -0.39 & 1.18 & $-10.59 * *$ & $-7.45 * *$ & -1.71 & $-3.05 * *$ & -1.15 & -1.30 & $-8.88 * *$ & $-3.65^{*}$ & -1.55 & 0.00 & $-3.39 * *$ & $-6.72 * *$ & -2.89 \\
\hline 30 & Bhuva-2 $x$ G.Til-1 & $-4.56 * *$ & 0.00 & $-11.79 * *$ & $-7.22 * *$ & -2.46 & $-4.20 * *$ & 0.77 & 0.43 & $-5.79 * *$ & -2.27 & $-2.71^{*}$ & $1.94^{*}$ & -1.69 & $-3.56 * *$ & -1.49 \\
\hline 31 & Bhuva-2 x G.Til-10 & $2.28 *$ & $6.08 * *$ & $-3.42 * *$ & -0.76 & $4.63^{* *}$ & $2.67 *$ & $6.90^{* *}$ & $9.96 * *$ & 0.77 & $4.94 * *$ & 4.26 ** & $8.14 * *$ & $7.63 * *$ & $3.16 * *$ & $5.77 * *$ \\
\hline 32 & Bhuva-2 x RT-54 & 1.59 & $3.17 * *$ & $-8.33 * *$ & $-1.98^{*}$ & -2.17 & $-2.29 *$ & -0.38 & 0.00 & $-4.63 * *$ & -1.88 & -0.78 & 0.78 & $-2.12^{*}$ & $-2.37 * *$ & -1.09 \\
\hline 33 & Khadkala-S x AT-285 & 0.40 & $5.24 * *$ & $-6.85 * *$ & 0.00 & -0.40 & $-4.96 * *$ & 0.00 & 0.00 & $-4.25 * *$ & -2.37 & $-3.49 * *$ & 1.16 & $-2.12^{*}$ & $-1.98 *$ & -1.59 \\
\hline 34 & Khadkala-S x G.Til-1 & $3.63 * *$ & $4.84 * *$ & $-6.85 * *$ & $2.42 * *$ & -0.69 & -1.91 & -0.38 & 0.00 & $-1.93^{*}$ & -1.09 & -0.39 & 0.78 & $-2.12^{*}$ & 0.40 & -0.30 \\
\hline 35 & Khadkala-S xG.Til-10 & $10.89 * *$ & $7.26 * *$ & $-2.02 *$ & $5.65 * *$ & $3.67 *$ & $4.96 * *$ & $1.92 *$ & $5.19^{* * *}$ & 1.16 & 3.26 & $6.59 * *$ & $3.10 * *$ & $2.97 * *$ & $3.56 * *$ & $4.08^{*}$ \\
\hline 36 & Khadkala-S x RT-54 & $6.85^{* *}$ & $3.63 * *$ & $-4.44 * *$ & 0.40 & -0.10 & 1.15 & -1.53 & $2.60^{*}$ & $-3.86 * *$ & -0.49 & $2.71^{*}$ & $\begin{array}{r}-0.39 \\
-10\end{array}$ & 0.42 & -1.58 & 0.30 \\
\hline 37 & IS-209 x AT-285 & 3.56 ** & $2.37^{*}$ & $-9.88 * *$ & $-2.77^{* *}$ & 0.20 & 0.00 & -0.77 & -1.30 & $-5.02 * *$ & -1.78 & 1.55 & 0.39 & $-3.39 * *$ & $-2.77^{* *}$ & -1.00 \\
\hline 38 & IS-209 x G.Til-1 & $3.56^{* * *}$ & $3.16^{* * *}$ & $-5.53 * *$ & $-1.98^{*}$ & 1.30 & 0.00 & 0.00 & $3.46^{* * *}$ & $-4.25 * *$ & -0.30 & 1.55 & 1.16 & 1.27 & $-1.98^{*}$ & 0.50 \\
\hline 39 & IS-209 x G.Til-10 & $8.70^{* * *}$ & $10.67 * *$ & $-2.77 * *$ & $4.74 * *$ & $6.92 * *$ & $4.96^{* * *}$ & $7.28 * *$ & $6.49^{* * *}$ & $2.32 * *$ & $5.23^{* *}$ & $6.59 * *$ & $8.53 * *$ & $4.24 * *$ & $4.74 * *$ & $6.07 * *$ \\
\hline \multirow[t]{4}{*}{40} & IS-209 x RT-54 & 0.79 & 0.79 & $-7.54 * *$ & $1.98^{*}$ & 0.10 & $-3.05^{* * *}$ & $-2.68 * *$ & 0.87 & -0.77 & -1.48 & -1.55 & -1.55 & -1.27 & 1.58 & -0.70 \\
\hline & Min. & -4.91 & -5.28 & -13.21 & -8.24 & -4.62 & -5.73 & -4.21 & -1.30 & -9.65 & -4.84 & -4.26 & -3.10 & -3.39 & -7.51 & -4.08 \\
\hline & Max. & 10.89 & 10.67 & -0.40 & 5.65 & 6.92 & 6.11 & 7.28 & 9.96 & 2.32 & 5.23 & 7.75 & 8.53 & 7.63 & 4.74 & 6.07 \\
\hline & Mean heterosis & 1.28 & 1.93 & -7.38 & -3.12 & -0.67 & -1.38 & -0.37 & 2.77 & -4.58 & -1.11 & 0.15 & 0.78 & 0.11 & -2.32 & -0.32 \\
\hline \multicolumn{2}{|c|}{ Desirable heterosis } & 5 & 6 & 39 & 27 & 1 & 20 & 14 & 0 & 35 & 4 & 9 & 5 & 11 & 25 & 1 \\
\hline
\end{tabular}


Table.4 Heterobeltiosis and standard heterosis over G.Til-2 and G.Til-3 under individual environments and pooled over environments for plant height $(\mathrm{cm})$

\begin{tabular}{|c|c|c|c|c|c|c|c|c|c|c|c|c|c|c|c|c|}
\hline \multirow{2}{*}{$\begin{array}{l}\text { Sr. } \\
\text { No. }\end{array}$} & \multirow[t]{2}{*}{ Crosses } & \multicolumn{5}{|c|}{ Heterobeltiosis $\left(\mathbf{H}_{1}\right)$} & \multicolumn{5}{|c|}{ Std. Heterosis over G.Til-2 $\left(\mathrm{H}_{2} \mathrm{C}_{1}\right)$} & \multicolumn{5}{|c|}{ Std. Heterosis over G.Til-3 $\left(\mathrm{H}_{2} \mathrm{C}_{2}\right)$} \\
\hline & & $\mathbf{E}_{1}$ & $\mathbf{E}_{2}$ & $\mathbf{E}_{3}$ & $\mathbf{E}_{4}$ & Pooled & $\mathbf{E}_{1}$ & $\mathbf{E}_{2}$ & $\mathbf{E}_{3}$ & $\mathbf{E}_{4}$ & Pooled & $\mathbf{E}_{1}$ & $\mathbf{E}_{2}$ & $\mathbf{E}_{3}$ & $\mathbf{E}_{4}$ & Pooled \\
\hline 1 & AT- $253 \times$ AT- 285 & $-3.82 *$ & $13.19^{* * *}$ & $-14.74 * *$ & $18.50^{* * *}$ & -1.04 & $-20.86^{* *}$ & $-16.73^{* * *}$ & $-22.0 * *$ & $-14.21 * *$ & $-18.18^{* *}$ & $-14.80^{* *}$ & $-9.36 * *$ & $-20.70 * *$ & $-12.47 * *$ & $-14.05^{* *}$ \\
\hline 2 & AT-253 x G.Til-1 & $7.28^{* *}$ & $13.37 * *$ & -1.61 & $17.72^{* * *}$ & 4.62 & $-11.73^{* *}$ & $-16.59^{* *}$ & $-9.99 * *$ & $-14.77^{* *}$ & $-13.50^{* *}$ & $-4.97 * *$ & $-9.22 * *$ & $-8.49 * *$ & $-13.05^{* *}$ & $-9.14 * *$ \\
\hline 3 & AT-253 x G.Til- 10 & $12.95 * *$ & $40.63^{* * *}$ & $12.53^{* *}$ & $37.65^{* * *}$ & $20.67 * *$ & $-7.07^{* *}$ & $3.47^{*}$ & 2.95 & -0.35 & -0.22 & 0.05 & $12.61 * *$ & $4.66^{* * *}$ & 1.67 & 4.80 \\
\hline 4 & AT- $-253 \times$ RT- 54 & $-4.36 * *$ & $3.76^{*}$ & $-10.08^{* *}$ & $-7.34 * *$ & $-8.50 *$ & -21.31 ** & $-23.66^{* * *}$ & $-17.74 * *$ & $-32.92 * *$ & $-24.34 * *$ & $-15.27 * *$ & $-16.91 * *$ & $-16.37^{* * *}$ & $-31.56^{* *}$ & $-20.53 * *$ \\
\hline 5 & AT- $265 \times$ AT- -285 & $-6.55 * *$ & $15.67 * *$ & $-4.22^{* *}$ & $7.46^{* * *}$ & 3.64 & $-19.49^{* *}$ & $-10.89^{* *}$ & $-8.24 * *$ & $-18.53^{* *}$ & $-14.48^{* *}$ & $-13.32^{* *}$ & $-3.01 *$ & $-6.71^{* *}$ & $-16.88^{* *}$ & $-10.17^{* *}$ \\
\hline 6 & AT-265 x G.Til-1 & 0.52 & $13.75^{* *}$ & $-4.78 * *$ & 11.97 *** & 4.90 & $-14.24 * *$ & $-13.21 * *$ & $-9.66^{* * *}$ & $-15.94 * *$ & $-13.44 * *$ & $-7.66 * *$ & $-5.54 * *$ & $-8.16^{* * *}$ & $-14.24 * *$ & $-9.07 * *$ \\
\hline 7 & AT-265 x G.Til-10 & $13.73^{* * *}$ & $23.76^{* * *}$ & -0.40 & $18.58^{* *}$ & $14.52 * *$ & -2.01 & $-4.65 * *$ & $-4.59 * *$ & $-10.11^{* *}$ & -5.50 & $5.50^{* *}$ & $3.77^{*}$ & -3.00 & $-8.29 * *$ & -0.73 \\
\hline 8 & AT-265 x RT-54 & $29.69 * *$ & $38.40^{* * *}$ & $18.46 * *$ & $31.57^{* * *}$ & $30.22 * *$ & $11.73^{* *}$ & $6.63 * *$ & $13.48^{* * *}$ & -0.26 & $7.46^{*}$ & $20.30^{* *}$ & $16.05 * *$ & $15.37 * *$ & 1.76 & $12.87^{* *}$ \\
\hline 9 & AT-306 x AT-285 & -5.50 ** & $10.57 * *$ & -0.60 & $19.44 * *$ & 3.91 & $-14.83^{* *}$ & $-10.89 * *$ & -0.38 & $-5.27 * *$ & $-8.02 * *$ & $-8.30^{* *}$ & $-3.01^{*}$ & 1.28 & $-3.35^{*}$ & -3.39 \\
\hline 10 & AT-306 x G.Til-1 & 1.09 & $6.85 * *$ & $-5.01 * *$ & $15.48^{* * *}$ & 0.44 & $-13.75^{* *}$ & $-18.48^{* * *}$ & $-9.88^{* * *}$ & $-13.3^{* *}$ & $-14.06^{* *}$ & $-7.14 * *$ & $-11.28 * *$ & $-8.38^{* * *}$ & $-11.55^{* *}$ & $-9.73 * *$ \\
\hline 11 & AT-306 x G.Til- 10 & $17.70^{* * *}$ & $36.06 * *$ & $20.75^{* * *}$ & $45.59 * *$ & $27.49 * *$ & $6.09 * *$ & $9.66 * *$ & $21.02 * *$ & $15.46^{* * *}$ & $12.85^{* *}$ & $14.22^{* *}$ & $19.35 * *$ & $23.03 * *$ & $17.81^{* *}$ & $18.53 * *$ \\
\hline 12 & AT- $306 \times$ RT- 54 & $14.43 * *$ & $23.75^{* * *}$ & $8.77^{* * *}$ & $19.34 * *$ & $14.30 * *$ & $3.14 *$ & -0.26 & $9.01^{* *}$ & $-5.36^{* *}$ & 1.17 & $11.05^{* *}$ & $8.55 * *$ & $10.82 * *$ & $-3.44 *$ & 6.27 \\
\hline 13 & AT-307 x AT-285 & $6.12 * *$ & $14.63 * *$ & 0.28 & $21.30^{* * *}$ & $10.6^{* * *}$ & $-6.33 * *$ & $-9.53 * *$ & -1.58 & $-5.79 * *$ & -6.02 & 0.85 & -1.53 & 0.06 & $-3.88 * *$ & -1.28 \\
\hline 14 & AT-307 x G.Til-1 & $10.87 * *$ & $18.07 * *$ & $5.75^{* *}$ & $21.98^{* * *}$ & $10.37^{* * *}$ & $-5.40 * *$ & $-9.92 * *$ & 0.33 & $-8.42^{* * *}$ & $-6.20^{*}$ & 1.85 & -1.96 & 2.00 & $-6.57 * *$ & -1.48 \\
\hline 15 & AT-307 x G.Til-10 & 2.45 & $22.97^{* * *}$ & $16.18 * *$ & $23.47^{* *}$ & $16.28 * *$ & $-9.57 * *$ & $-2.94 *$ & $14.03^{* * *}$ & $-4.10^{* * *}$ & -1.18 & -2.64 & $5.64^{* *}$ & $15.93 * *$ & -2.16 & 3.80 \\
\hline 16 & AT- $307 \times$ RT- 54 & $13.52 * *$ & $20.75^{* *}$ & $9.18^{* *}$ & $19.58^{* * *}$ & $15.77^{* * *}$ & 0.20 & $-4.70^{* * *}$ & $7.15^{* *}$ & $-7.13^{* * *}$ & -1.62 & $7.88^{* * *}$ & $3.73^{*}$ & $8.93^{* * *}$ & $-5.24 * *$ & 3.34 \\
\hline 17 & AT-319 x AT-285 & $10.13^{* * *}$ & $18.10^{* * *}$ & $4.80^{* * *}$ & $21.44 * *$ & $9.33^{*}$ & -7.71 ** & $-11.50^{* * *}$ & -2.35 & $-10.45^{* * *}$ & $-8.32 * *$ & -0.63 & $-3.68^{*}$ & -0.72 & $-8.64 * *$ & -3.70 \\
\hline 18 & AT-319 x G.Til-1 & $-21.50 * *$ & $23.43 * *$ & $-14.06 * *$ & $21.56^{* * *}$ & -1.51 & $-34.22 * *$ & $-7.51^{* *}$ & $-19.92 * *$ & $-10.37 * *$ & $-17.41^{* *}$ & $-29.18^{* * *}$ & 0.67 & -18.59 ** & -8.55 ** & $-13.24 * *$ \\
\hline 19 & AT-319 x G.Til-10 & $17.16^{* * *}$ & $26.48^{* * *}$ & $9.90^{* *}$ & $19.74 * *$ & $13.85^{* * *}$ & -1.82 & -5.22 *** & 2.40 & $-11.71 * *$ & -4.53 & $5.71^{* *}$ & $3.15^{*}$ & $4.11^{* * *}$ & $-9.92 * *$ & 0.29 \\
\hline 20 & AT- $319 \times$ RT-54 & $-25.37 * *$ & 23.84 ** & $-25.37 * *$ & $26.48^{* * *}$ & -3.88 & $-37.46 * *$ & $-7.20 * *$ & $-30.46 * *$ & $-6.74 * *$ & $-19.39 * *$ & $-32.66 * *$ & 1.00 & -29.30 ** & -4.85 ** & $-15.33^{* *}$ \\
\hline 21 & AT-322 x AT-285 & -19.81 *** & $9.85^{* * *}$ & $-22.97 * *$ & $8.27 * *$ & $-7.26^{*}$ & $-25.28 * *$ & -8.47 ** & $-20.20 * *$ & $-11.23 * *$ & $-15.81 * *$ & $-19.56^{* *}$ & -0.38 & $-18.87 * *$ & $-9.43 * *$ & $-11.57^{* *}$ \\
\hline 22 & AT-322 x G.Til-1 & $-13.29 * *$ & $9.84 * *$ & $-6.33 * *$ & $13.29^{* *}$ & -3.14 & $-26.02 * *$ & $-16.20^{* *}$ & $-11.14 * *$ & $-14.95^{* * *}$ & $-17.12^{* *}$ & $-20.35^{* * *}$ & $-8.79 * *$ & $-9.66 * *$ & $-13.22 * *$ & $-12.95 * *$ \\
\hline 23 & AT-322 x G.Til-10 & $-7.80 * *$ & $14.70^{* * *}$ & $3.27 *$ & $18.49^{* * *}$ & 5.91 & $-14.09 * *$ & $-4.43 * *$ & $6.99 * *$ & $-2.85^{*}$ & -3.85 & $-7.51^{* *}$ & $4.01^{* *}$ & $8.77 * *$ & -0.88 & 0.99 \\
\hline 24 & AT-322 x RT-54 & $-24.93 * *$ & $12.47 * *$ & $-12.15 * *$ & $14.50^{* * *}$ & -5.14 & $-31.12^{* *}$ & $-7.73 * *$ & $-10.37 * *$ & $-7.56^{* * *}$ & $-13.89 * *$ & $-25.85^{* *}$ & 0.43 & $-8.88 * *$ & $-5.69^{* *}$ & $-9.55^{* * *}$ \\
\hline 25 & AT-341 × AT-285 & $12.37 * *$ & $16.95^{* * *}$ & $7.55^{* *}$ & $9.41^{* * *}$ & $9.05 * *$ & $9.67 * *$ & 2.06 & $16.70^{* * *}$ & $-6.05^{* *}$ & 4.85 & $18.08^{* *}$ & $11.08 * *$ & $18.65 * *$ & $-4.14^{* *}$ & $10.13^{* *}$ \\
\hline 26 & AT-341 x G.Til-1 & $9.90 * *$ & $15.42 * *$ & $5.70 * *$ & $4.32^{*}$ & 4.50 & $-6.23 * *$ & $-11.94 * *$ & 0.27 & $-21.68^{* * *}$ & $-10.59 * *$ & 0.95 & $-4.16 * *$ & 1.94 & $-20.10^{* *}$ & -6.08 \\
\hline 27 & AT-341 x G.Til-10 & $-2.93^{*}$ & $7.74 * *$ & $-10.75 * *$ & 1.34 & -2.97 & 13.89 ** & $13.04 * *$ & $16.43^{* * *}$ & $4.62^{* * *}$ & $11.68^{* * *}$ & $22.62^{* *}$ & $23.03 * *$ & $18.37 * *$ & $6.74 * *$ & $17.3^{* *}$ \\
\hline 28 & AT- $341 \times$ RT-54 & $10.01 * *$ & 13.59 ** & $5.51^{* *}$ & $5.83^{* * *}$ & -3.36 & 0.93 & $-6.80 * *$ & $7.64 * *$ & $-14.56^{* * *}$ & -3.94 & $8.67 * *$ & 1.43 & $9.43 * *$ & $-12.83 * *$ & 0.91 \\
\hline 29 & Bhuva-2 $\times$ AT-285 & $51.48^{* *}$ & $54.02^{* * *}$ & $55.03 * *$ & $57.78^{* * *}$ & $20.40^{* * *}$ & 2.70 & $-6.63 * *$ & $16.87^{* * *}$ & $-5.87 * *$ & 0.91 & $10.57 * *$ & 1.62 & $18.81^{* * *}$ & $-3.97 * *$ & 6.00 \\
\hline 30 & Bhuva-2 x G.Til-1 & $-7.68 * *$ & $51.12 * *$ & $28.46 * *$ & $59.09 * *$ & 3.44 & $-37.41 * *$ & $-8.38 * *$ & $-3.17^{*}$ & $-5.10^{* * *}$ & $-13.34 * *$ & $-32.61 * *$ & -0.29 & -1.55 & $-3.17^{*}$ & $-8.97 * *$ \\
\hline 31 & Bhuva-2 x G.Til-10 & $51.19^{* * *}$ & $82.40^{* * *}$ & 67.34 ** & $83.71^{* * *}$ & $33.40 * *$ & 2.50 & 10.58 ** & $26.15^{* * *}$ & $9.59^{* * *}$ & $11.73^{* *}$ & $10.36^{* *}$ & $20.30^{* *}$ & $28.25 * *$ & $11.81^{* * *}$ & $17.37^{* * *}$ \\
\hline 32 & Bhuva-2 x RT-54 & $6.37 * *$ & $39.91^{* * *}$ & $-7.24 * *$ & $49.02^{* * *}$ & -4.92 & $-27.88 * *$ & $-15.18 * *$ & $-30.08 * *$ & $-11.10^{* *}$ & $-20.35 * *$ & $-22.36^{* *}$ & $-7.69 * *$ & $-28.91 * *$ & $-9.30^{* *}$ & $-16.33 * *$ \\
\hline 33 & Khadkala-S xAT285 & $-6.54 * *$ & -2.67 & $-17.40^{* *}$ & $-4.78^{* *}$ & $-9.93 * *$ & $-8.79 * *$ & $-15.06^{* * *}$ & $-10.37 * *$ & $-18.23 * *$ & $-13.40^{* *}$ & -1.80 & $-7.55^{* *}$ & $-8.88^{* *}$ & $-16.57^{* *}$ & $-9.04 * *$ \\
\hline 34 & Khadkala-S x G.Till & $-10.41^{* *}$ & $20.60^{* * *}$ & $9.21^{* *}$ & $23.48^{* * *}$ & 6.31 & $-23.56^{* *}$ & -7.99 ** & $3.60^{*}$ & $-7.30^{* *}$ & $-9.04^{* *}$ & $-17.71^{* *}$ & 0.14 & $5.33^{* *}$ & $-5.42 * *$ & -4.46 \\
\hline 35 & Khadkala-S xGTil10 & $-17.78^{* * *}$ & $3.60^{* *}$ & $-27.15 * *$ & $10.67 * *$ & $-8.94 * *$ & $-3.53^{*}$ & $8.69 * *$ & $-4.97 * *$ & $14.25 * *$ & 4.31 & $3.86^{* * *}$ & $18.30^{* *}$ & $-3.39^{*}$ & $16.57 * *$ & $9.57^{* *}$ \\
\hline 36 & Khadkala-S x RT-54 & $7.92^{* * *}$ & $12.68^{* * *}$ & -2.94 & $21.62^{* * *}$ & -2.40 & -0.98 & $-7.55^{* *}$ & -0.98 & -1.81 & -2.98 & $6.61^{* *}$ & 0.62 & 0.67 & 0.18 & 1.91 \\
\hline 37 & IS-209 x AT-285 & $9.84 * *$ & $33.84 * *$ & $19.68 * *$ & 44.61 ** & $9.03^{*}$ & $-18.90^{* * *}$ & $-11.63 * *$ & -1.75 & $-6.05 * *$ & $-9.71^{* * *}$ & $-12.68 * *$ & $-3.82^{*}$ & -0.11 & $-4.14 * *$ & -5.16 \\
\hline 38 & IS-209 x G.Til-1 & $-13.36 * *$ & $37.43 * *$ & $-9.77 * *$ & 47.61 ** & -0.86 & $-36.03 * *$ & $-9.26^{* * *}$ & $-25.93 * *$ & $-4.10^{* * *}$ & $-17.90 * *$ & $-31.13 * *$ & -1.24 & $-24.69 * *$ & -2.16 & $-13.77 * *$ \\
\hline 39 & IS-209 x G.Til-10 & $53.92 * *$ & $65.03 * *$ & 56.38 ** & $67.62^{* * *}$ & $38.00^{* * *}$ & $13.65^{* * *}$ & $8.96^{* *}$ & $28.38 * *$ & $8.90^{* * *}$ & $14.28 * *$ & $22.36^{* *}$ & $18.60^{* *}$ & $30.52 * *$ & $11.11^{* *}$ & $20.03 * *$ \\
\hline \multirow[t]{4}{*}{40} & IS-209 x RT-54 & $-9.51^{* * *}$ & $27.59^{* *}$ & $14.89 * *$ & $16.42 * *$ & -3.54 & $-33.19^{* *}$ & $-15.76 * *$ & $-5.68 * *$ & $-24.36^{* * *}$ & $-20.13^{* *}$ & $-28.07^{* * *}$ & $-8.31 * *$ & $-4.11 * *$ & $-22.83^{* *}$ & $-16.1 * *$ \\
\hline & Min. & -25.37 & -2.67 & -27.15 & -7.34 & -9.93 & -37.46 & -23.66 & -30.46 & -32.92 & -24.34 & -32.66 & -16.91 & -29.30 & -31.56 & -20.53 \\
\hline & Max. & 53.92 & 82.40 & 67.34 & 83.71 & 38.00 & 13.89 & 13.04 & 28.38 & 15.46 & 14.28 & 22.62 & 23.03 & 30.52 & 17.81 & 20.03 \\
\hline & Mean heterosis & 4.14 & 23.35 & 4.55 & 24.09 & 6.59 & -11.41 & -6.60 & -1.70 & -7.64 & -6.98 & -4.62 & 1.66 & -0.06 & -5.77 & -2.29 \\
\hline Jes & & 20 & & & & & & & & & & & & 15 & & \\
\hline
\end{tabular}


Table.5 Heterobeltiosis and standard heterosis over G.Til-2 and G.Til-3 under individual environments and pooled over environments for height to first capsule

\begin{tabular}{|c|c|c|c|c|c|c|c|c|c|c|c|c|c|c|c|c|}
\hline \multirow{2}{*}{$\begin{array}{l}\text { Sr. } \\
\text { No. }\end{array}$} & \multirow[t]{2}{*}{ Crosses } & \multicolumn{5}{|c|}{ Heterobeltiosis $\left(\mathbf{H}_{1}\right)$} & \multicolumn{5}{|c|}{ Std. Heterosis over G.Til-2 $\left(\mathrm{H}_{2} \mathrm{C}_{1}\right)$} & \multicolumn{5}{|c|}{ Std. Heterosis over G.Til-3 $\left(\mathrm{H}_{2} \mathrm{C}_{2}\right)$} \\
\hline & & $\mathbf{E}_{1}$ & $\mathbf{E}_{2}$ & $\mathbf{E}_{3}$ & $\mathbf{E}_{4}$ & Pooled & $\mathbf{E}_{1}$ & $\mathbf{E}_{2}$ & $\mathbf{E}_{3}$ & $\mathbf{E}_{4}$ & Pooled & $E_{1}$ & $\mathbf{E}_{2}$ & $\mathbf{E}_{3}$ & $\mathbf{E}_{4}$ & Pooled \\
\hline 1 & AT-253 x AT-285 & $-36.02 * *$ & 13.38 ** & $-40.14 * *$ & 30.36 ** & -14.86 & $-53.49 * *$ & $-31.57 * *$ & $-47.28 * *$ & $-22.84 * *$ & $-37.25 * *$ & $-43.83 * *$ & $-20.93 * *$ & $-42.79 * *$ & $-20.17 * *$ & $-30.01 * * *$ \\
\hline 2 & AT-253 x G.Til-1 & 1.54 & $14.92 * *$ & -3.95 & $35.16^{* * *}$ & 3.69 & $-26.18 * *$ & $-30.64 * *$ & $-15.41^{* *}$ & $-20.00 * *$ & $-23.57 * *$ & $-10.84 * *$ & $-19.86^{* * *}$ & $-8.20^{*}$ & $-17.23^{* *}$ & $-14.76^{*}$ \\
\hline 3 & AT- $253 \times$ G.Til- 10 & 6.17 & 77.19 ** & -6.52 & $81.82^{* * *}$ & 29.40 ** & $-22.82 * *$ & $6.94 *$ & $-17.67 * *$ & $7.61 * *$ & -4.63 & -6.78 & $23.56^{* * *}$ & $-10.66 * *$ & $11.34^{* *}$ & 6.37 \\
\hline 4 & AT- $253 \times$ RT-54 & -7.89 & 1.37 & $-26.93 * *$ & 5.15 & -13.91 & $-33.04 * *$ & $-38.82 * *$ & $-35.65^{* *}$ & $-37.77 * *$ & $-36.54 * *$ & $-19.13^{* *}$ & $-29.31 * *$ & $-30.16^{* *}$ & -35.61 ** & $-29.23 * * *$ \\
\hline 5 & AT-265 x AT-285 & $-15.97 * *$ & 4.03 & $-25.97 * *$ & -4.03 & -13.92 & $-35.04 * *$ & $-33.23 * *$ & $-30.66^{* * *}$ & $-39.59 * *$ & $-34.99 * *$ & $-21.54 * *$ & $-22.85 * *$ & $-24.75 * *$ & $-37.5^{* *}$ & $-27.5 * *$ \\
\hline 6 & AT-265 x G.Til-1 & 4.68 & 38.13 ** & 0.67 & $52.84 * *$ & $15.08^{*}$ & $-21.95 * *$ & $-14.49 * *$ & $-9.06 * *$ & $-7.21^{*}$ & $-13.09 *$ & -5.72 & -1.20 & -1.31 & -3.99 & -3.07 \\
\hline 7 & AT-265 x G.Til-10 & $15.81^{* *}$ & $51.13 * *$ & -5.97 & $56.77^{* * *}$ & $24.47 * *$ & $-10.47 * *$ & -3.00 & $-11.93 * *$ & -1.32 & -6.00 & $8.13 *$ & $12.08^{* * *}$ & -4.43 & 2.10 & 4.83 \\
\hline 8 & AT-265 x RT-54 & $53.71 * *$ & $78.55^{* * *}$ & $37.10^{* * *}$ & $57.58^{* * *}$ & $50.72 * *$ & $18.83 * *$ & $14.60^{* *}$ & 28.40 ** & -0.81 & $13.82^{*}$ & $43.52^{* * *}$ & $32.42 * *$ & $39.34 * *$ & 2.63 & $26.94 * *$ \\
\hline 9 & AT-306 x AT-285 & $-19.22 * *$ & 45.47 ** & $-15.94 * *$ & $60.63^{* * *}$ & 12.09 & $-35.54 * *$ & -3.62 & $-18.73^{* * *}$ & 4.37 & $-11.74 *$ & $-22.14 * *$ & $11.36^{* *}$ & $-11.80^{* *}$ & $7.98 * *$ & -1.57 \\
\hline 10 & AT-306 x G.Til-1 & $37.29 * *$ & $54.85^{* * *}$ & $25.08 * *$ & $75.25 * *$ & 31.76 ** & 2.37 & -4.14 & 12.99 ** & $6.40^{*}$ & 3.75 & $23.64 * *$ & 10.77 ** & $22.62 * *$ & $10.08 * *$ & $15.71 *$ \\
\hline 11 & AT-306 x G.Til-10 & $11.88 * *$ & $99.38 * *$ & $7.50^{*}$ & $110.94 * *$ & $49.87 * *$ & $-10.72 * *$ & $32.09 * *$ & 3.93 & $37.06 * *$ & $18.01 * *$ & $7.83^{*}$ & $52.63 * *$ & $12.79 * *$ & $41.81 * *$ & $31.61 * *$ \\
\hline 12 & AT-306 x RT-54 & $44.38 * *$ & 69.22*** & $26.41^{* * *}$ & $47.34 * *$ & $39.79 * *$ & $15.21 * *$ & $12.11 * *$ & 22.21 ** & -4.26 & 10.07 & $39.16^{* *}$ & $29.55 * *$ & $32.62 * *$ & -0.95 & $22.76 * *$ \\
\hline 13 & AT-307 x AT-285 & $27.93 * *$ & $50.91^{\text {** }}$ & $17.02 * *$ & $68.43 * *$ & 33.26 ** & -3.49 & -5.49 & $6.95^{*}$ & 3.45 & -0.03 & $16.57 * *$ & $9.21 * *$ & $16.07 * *$ & $7.04 *$ & 11.50 \\
\hline 14 & AT-307 x G.Til-1 & $23.24 * *$ & $42.81^{* *}$ & $13.21 * *$ & $57.53^{* * *}$ & 25.29 ** & $-8.10 * *$ & $-11.59 * *$ & 2.27 & -4.37 & -6.00 & $10.99 * *$ & 2.15 & $10.98 * *$ & -1.05 & 4.83 \\
\hline 15 & AT-307 x G.Til-10 & 19.67 ** & $67.11^{* *}$ & 10.41 ** & $68.93 * *$ & 33.68 ** & $-9.73 * *$ & 4.66 & 0.91 & 3.76 & 0.29 & $9.04 *$ & $20.93 * *$ & $9.51^{*}$ & $7.35^{*}$ & 11.85 \\
\hline 16 & AT- $307 \times$ RT-54 & $43.47^{* * *}$ & $68.76^{* * *}$ & $32.07 * *$ & $67.93^{* * *}$ & 44.57 *** & $8.23 * *$ & 5.69 & $20.69 * *$ & 3.15 & 8.46 & $30.72^{* *}$ & $22.13 * *$ & $30.98 * *$ & $6.72^{*}$ & $20.97 * *$ \\
\hline 17 & AT-319 $\times$ AT-285 & $24.64^{* * *}$ & $43.92^{* * *}$ & 13.29 ** & $47.16^{* *}$ & $26.07 * *$ & -4.11 & $-8.07 * *$ & 5.59 & $-7.82 * *$ & -4.42 & $15.81^{* *}$ & 6.22 & 14.59 ** & -4.62 & 6.60 \\
\hline 18 & AT-319 x G.Til-1 & $-38.63^{* * *}$ & $54.68^{* * *}$ & $-27.59 * *$ & $55.52^{* * *}$ & 2.55 & $-54.24 * *$ & -4.24 & $-34.59^{* *}$ & -5.58 & $-22.25 * *$ & $-44.73^{* *}$ & $10.65^{* * *}$ & $-29.02 * *$ & -2.31 & $-13.29 *$ \\
\hline 19 & AT-319 x G.Til-10 & $30.63 * *$ & $49.92 * *$ & $-15.72 * *$ & 33.23 ** & $18.69^{*}$ & 0.50 & -4.24 & $-21.45^{* *}$ & $-16.55^{* * *}$ & -10.01 & $21.39^{* *}$ & $10.65^{* * *}$ & $-14.75^{* *}$ & $-13.66^{* *}$ & 0.36 \\
\hline 20 & AT-319 x RT-54 & $-36.3 * *$ & $45.06 * *$ & $-43.11 * *$ & $60.78^{* * *}$ & 1.62 & $-51.0^{* *}$ & $-7.35 *$ & $-46.98^{* * *}$ & 0.71 & $-22.96 * *$ & $-40.81 * *$ & $7.06^{*}$ & $-42.46 * *$ & 4.20 & $-14.08 *$ \\
\hline 21 & AT- $322 \times$ AT-285 & $-29.87^{* * *}$ & 27.49 ** & $-34.62 * *$ & $42.64 * *$ & -4.18 & $-41.15 * *$ & $-11.18^{* * *}$ & $-33.53 * *$ & -2.54 & $-20.06 * *$ & $-28.92 * *$ & 2.63 & $-27.87 * *$ & 0.84 & -10.84 \\
\hline 22 & AT- $322 \times$ G.Til- 1 & $-27.93 * *$ & $30.77 * *$ & $-25.75 * *$ & $42.64 * *$ & -8.59 & $-46.26 * *$ & $-19.05 * *$ & $-32.93 * *$ & $-13.40^{* * *}$ & $-26.50^{* *}$ & $-35.09 * *$ & -6.46 & $-27.21 * *$ & $-10.40 * *$ & $-18.03 * *$ \\
\hline 23 & AT- $322 \times$ G.Til- 10 & $-15.45^{* *}$ & 44.13*** & $-14.26 * *$ & $43.09 * *$ & 8.07 & $-29.05 * *$ & 0.41 & $-12.84^{* * *}$ & -2.23 & -9.84 & $-14.31^{* *}$ & $16.03 * *$ & -5.41 & 1.16 & 0.56 \\
\hline 24 & AT- $322 \times$ RT-54 & $-43.24^{* * *}$ & $31.5 * *$ & $-28.97 * *$ & $44.13 * *$ & -4.70 & $-52.37 * *$ & $-8.39 * *$ & $-27.79 * *$ & -1.52 & $-20.50^{* *}$ & $-42.47 * *$ & 5.86 & $-21.64 * *$ & 1.89 & -11.33 \\
\hline 25 & AT-341 x AT-285 & $-47.71 * *$ & $12.19^{* * *}$ & $-44.43 * *$ & $18.74 * *$ & $-18.27 * *$ & $-50.25 * *$ & $-11.39 * *$ & $-35.95 * *$ & $-8.02 * *$ & $-24.30^{* * *}$ & $-39.91 * *$ & 2.39 & $-30.49 * *$ & -4.83 & $-15.58^{*}$ \\
\hline 26 & AT-341 x G.Til-1 & $28.09 * *$ & $37.79 * *$ & $17.22 * *$ & $23.75^{* * *}$ & 10.38 & -4.49 & $-14.70^{* * *}$ & 5.89 & $-24.87 * *$ & -11.24 & $15.36^{* *}$ & -1.44 & $14.92 * *$ & $-22.27 * *$ & -1.01 \\
\hline 27 & AT-341 x G.Til-10 & $-20.21 * *$ & $-13.44 * *$ & $-42.00 * *$ & $5.80^{*}$ & $-22.66 * *$ & $13.22 * *$ & 1.97 & -0.30 & $22.23 * *$ & 10.01 & $36.75 * *$ & $17.82^{* * *}$ & $8.20^{*}$ & $26.47 * *$ & $22.70^{* * *}$ \\
\hline 28 & AT- $341 \times$ RT-54 & $10.84^{* *}$ & $15.72^{* * *}$ & 0.95 & $13.01 * *$ & -9.19 & 2.00 & $-11.59 * *$ & $12.54 * *$ & $-15.33 * *$ & -4.80 & $23.19 * *$ & 2.15 & $22.13 * *$ & $-12.39 * *$ & 6.17 \\
\hline 29 & Bhuva-2 $x$ AT-285 & $98.60 * *$ & $160.96 * *$ & $82.58 * *$ & $140.73 * *$ & $20.70^{* * *}$ & $-11.85 * *$ & -3.83 & -1.81 & $-12.99 * *$ & -7.96 & 6.48 & $11.12 * *$ & 6.56 & $-9.98 * *$ & 2.65 \\
\hline 30 & Bhuva-2 x G.Til-1 & -9.83 & $125.84 * *$ & $36.52 * *$ & $170.22 * *$ & -1.19 & $-59.98 * *$ & $-16.77 * *$ & $-26.59 * *$ & -2.34 & $-24.66 * *$ & $-51.66^{* * *}$ & -3.83 & $-20.33 * *$ & 1.05 & $-15.97 *$ \\
\hline 31 & Bhuva-2 x G.Til-10 & $114.30^{\text {*** }}$ & 256.20 *** & $103.90^{* * *}$ & $241.85 * *$ & 52.61 ** & -4.86 & $31.26^{* *}$ & $9.67 * *$ & $23.55 * *$ & $16.37^{* * *}$ & $14.91^{* *}$ & 51.67 *** & $19.02 * *$ & $27.84 * *$ & $29.78^{* *}$ \\
\hline 32 & Bhuva-2 $\times$ RT-54 & $24.16 * *$ & 98.31 ** & -1.40 & $144.94 * *$ & -8.95 & $-44.89 * *$ & $-26.92 * *$ & $-46.98 * *$ & $-11.47 * *$ & $-30.57 * *$ & $-33.43 * *$ & $-15.55 * *$ & $-42.46 * *$ & $-8.4 * *$ & $-22.57 * *$ \\
\hline 33 & Khadkala-S xAT285 & $-8.13^{*}$ & -2.49 & $-32.63 * *$ & -1.97 & $-14.42 *$ & $-12.59 * *$ & $-22.98 * *$ & $-22.36 * *$ & $-24.06 * *$ & $-20.73 * *$ & 5.57 & $-11.00^{* * *}$ & $-15.74 * *$ & $-21.43^{* *}$ & -11.59 \\
\hline 34 & Khadkala-S xG.Til-1 & $-21.57^{* * *}$ & $36.62 * *$ & 7.53 & $40.64 * *$ & 0.87 & $-41.52^{* * *}$ & $-15.42 * *$ & -2.87 & $-14.62^{* * *}$ & $-18.89 * *$ & $-29.37 * *$ & -2.27 & 5.41 & $-11.66 * *$ & -9.54 \\
\hline 35 & Khadkala-S xGTil10 & $-42.25 * *$ & $14.12^{* * *}$ & $-48.12 * *$ & $29.15 * *$ & $-17.96 * *$ & $-21.45 * *$ & $28.88 * *$ & $-14.50^{* * *}$ & $43.05^{* * *}$ & $12.74 *$ & -5.12 & $48.92 * *$ & -7.21 & 48.00 ** & $25.73^{* *}$ \\
\hline 36 & Khadkala-S x RT-54 & $9.89 * *$ & 18.16 ** & $-23.71^{* *}$ & $29.00 * *$ & -10.67 & 1.12 & $-9.73 * *$ & $-14.95 * *$ & -3.35 & -6.35 & $22.14^{* * *}$ & 4.31 & $-7.70^{*}$ & 0.00 & 4.44 \\
\hline 37 & IS-209 x AT-285 & 8.77 & $83.33^{* * *}$ & 6.80 & $80.26 * *$ & 16.60 & $-38.15 * *$ & $-13.46^{* * *}$ & $-26.44 * *$ & $-16.55^{* * *}$ & $-22.66^{* *}$ & $-25.30^{* *}$ & 0.00 & $-20.16^{* * *}$ & $-13.66 * *$ & $-13.75^{*}$ \\
\hline 38 & IS-209 x G.Til-1 & $-23.68 * *$ & $95.83 * *$ & -7.46 & $115.79 * *$ & 16.87 & $-56.61 * *$ & $-7.56^{*}$ & $-36.25^{* * *}$ & -0.10 & $-22.49 * *$ & $-47.59 * *$ & $6.82 *$ & $-30.82 * *$ & 3.36 & $-13.55^{*}$ \\
\hline 39 & IS-209 x G.Til-10 & $72.81^{* *}$ & $193.42^{* * *}$ & $104.61 * *$ & $166.45^{* *}$ & 88.70 *** & -1.75 & $38.51 * *$ & $40.94 * *$ & $23.35 * *$ & $25.15 * *$ & 18.67 ** & $60.05^{* * *}$ & $52.95 * *$ & $27.63^{* * *}$ & $39.58 * *$ \\
\hline 40 & IS-209 x RT-54 & $-11.84^{*}$ & $60.53 * *$ & $12.94 *$ & $57.24 * *$ & 4.46 & $-49.88^{* *}$ & $-24.22 * *$ & $-22.21^{* * *}$ & $-27.21^{* * *}$ & $-30.72 * *$ & $-39.46 * *$ & $-12.44 * *$ & $-15.57 * *$ & $-24.68 * *$ & $-22.73 * *$ \\
\hline & Min. & -47.71 & -13.44 & -48.12 & -4.03 & -22.66 & -59.98 & -38.82 & -47.28 & -39.59 & -37.25 & -51.66 & -29.31 & -42.79 & -37.50 & -30.01 \\
\hline & Max. & 114.33 & 256.18 & 104.61 & 241.85 & 88.70 & 18.83 & 38.51 & 40.94 & 43.05 & 25.15 & 43.52 & 60.05 & 52.95 & 48.00 & 39.58 \\
\hline & Mean heterosis & 6.42 & 57.14 & 1.02 & 62.34 & 12.36 & -22.14 & -6.01 & -11.87 & -4.25 & -10.43 & -5.96 & 8.60 & -4.36 & -0.93 & -0.10 \\
\hline Desi & le heterosis & 16 & 1 & 16 & 0 & 4 & 27 & 22 & 24 & 17 & 19 & 18 & 7 & 20 & 14 & 13 \\
\hline
\end{tabular}


Table.6 Heterobeltiosis and standard heterosis over G.Til-2 and G.Til-3 under individual environments and pooled over environments for seed yield per plant $(\mathrm{g})$

\begin{tabular}{|c|c|c|c|c|c|c|c|c|c|c|c|c|c|c|c|c|}
\hline \multirow{2}{*}{$\begin{array}{l}\text { Sr. } \\
\text { No. }\end{array}$} & \multirow[t]{2}{*}{ Crosses } & \multicolumn{5}{|c|}{ Heterobeltiosis $\left(\mathbf{H}_{1}\right)$} & \multicolumn{5}{|c|}{ Std. Heterosis over G.Til-2 $\left(\mathrm{H}_{2} \mathrm{C}_{1}\right)$} & \multicolumn{5}{|c|}{ Std. Heterosis over G.Til-3 $\left(\mathrm{H}_{2} \mathrm{C}_{2}\right)$} \\
\hline & & $\mathbf{E}_{1}$ & $\mathbf{E}_{2}$ & $\mathbf{E}_{3}$ & $\mathbf{E}_{4}$ & Pooled & $\mathbf{E}_{1}$ & $\mathbf{E}_{2}$ & $\mathbf{E}_{3}$ & $\mathbf{E}_{4}$ & Pooled & $\mathbf{E}_{1}$ & $\mathbf{E}_{2}$ & $\mathbf{E}_{3}$ & $\mathbf{E}_{4}$ & Pooled \\
\hline 1 & AT-253 $\times$ AT- 285 & $-22.92^{*}$ & $63.19^{* *}$ & $-20.83 *$ & $20.14^{*}$ & 8.39 & $-36.21^{* *}$ & 12.44 & $-17.99^{*}$ & -8.95 & -11.10 & $-45.59^{* *}$ & $-11.99 *$ & $-31.74 * *$ & $-19.16^{* *}$ & $-25.70^{* *}$ \\
\hline 2 & AT-253 x G.Til-1 & $-44.37 * *$ & $62.25^{* *}$ & $-45.70 * *$ & $17.88^{*}$ & -3.13 & $-51.72 * *$ & $17.22^{*}$ & $-41.01 * *$ & -6.32 & $-17.28 *$ & $-58.82^{* *}$ & -8.24 & $-50.9^{* *}$ & $-16.82^{* * *}$ & $-30.87 * *$ \\
\hline 3 & AT-253 x G.Til-10 & -1.60 & $48.40^{* *}$ & $-33.20^{* *}$ & $-40.80^{* *}$ & 6.76 & $41.38^{* *}$ & $77.51^{* *}$ & $20.14 *$ & $-22.11 * *$ & $30.90^{* *}$ & $20.59^{* *}$ & $38.95^{* *}$ & 0.00 & $-30.84 * *$ & 9.39 \\
\hline 4 & AT- $253 \times$ RT-54 & $36.61^{* * *}$ & $-25.68^{* *}$ & $-28.96 * *$ & -7.10 & -12.83 & $43.68^{* * *}$ & $-34.93 * *$ & -6.47 & -10.53 & -3.65 & $22.55 * *$ & $-49.06 * *$ & $-22.16^{* * *}$ & $-20.56^{* * *}$ & $-19.48^{* *}$ \\
\hline 5 & AT-265 x AT-285 & $-19.58^{*}$ & -3.50 & $-32.17 * *$ & -11.89 & -7.57 & $-33.91^{* *}$ & $-33.97 * *$ & $-30.22^{* *}$ & $-33.68^{* *}$ & $-33.15^{* * *}$ & $-43.63^{* * *}$ & $-48.31 * *$ & $-41.92^{* *}$ & $-41.12 * *$ & $-44.13^{* *}$ \\
\hline 6 & AT-265 x G.Til-1 & -15.89 & -19.21 & -8.61 & 2.65 & -10.86 & $-27.01 * *$ & $-41.63 * *$ & -0.72 & $-18.42^{* *}$ & $-23.88^{* * *}$ & $-37.75^{* * *}$ & $-54.31 * *$ & $-17.37^{*}$ & $-27.57 * *$ & $-36.38 * *$ \\
\hline 7 & AT-265 x G.Til-10 & -0.80 & $58.40^{* * *}$ & $-53.60^{* * *}$ & -46.80 ** & 2.29 & $42.53^{* *}$ & $89.47 * *$ & -16.55 & $-30.00 * *$ & $25.42 * *$ & $21.57 * *$ & $48.31 * *$ & $-30.54 * *$ & $-37.85^{* *}$ & 4.81 \\
\hline 8 & AT-265 x RT-54 & $-42.08 * *$ & 8.74 & $-45.90 * *$ & $-44.26^{6 * *}$ & $-35.71^{* *}$ & $-39.08^{* * *}$ & -4.78 & $-28.78 * *$ & $-46.32 * *$ & $-28.93 * *$ & $-48.04 * *$ & $-25.47 * *$ & $-40.72^{* * *}$ & $-52.34 * *$ & $-40.61 * *$ \\
\hline 9 & AT-306 x AT-285 & 18.88 & $32.87^{* *}$ & 2.10 & -17.48 & $21.17^{*}$ & -2.30 & -9.09 & 5.04 & $-37.89 * *$ & -12.36 & $-16.67^{*}$ & $-28.84 * *$ & -12.57 & $-44.86 * *$ & $-26.76^{* * *}$ \\
\hline 10 & AT-306 x G.Til-1 & $-33.77^{* * *}$ & 13.25 & $-34.44 * *$ & -5.30 & -15.63 & $-42.53^{* *}$ & $-18.18^{*}$ & $-28.78^{* * *}$ & $-24.74 * *$ & $-27.95^{* * *}$ & $-50.98 * *$ & $-35.96 * *$ & $-40.72 * *$ & $-33.18 * *$ & $-39.79 * *$ \\
\hline 11 & AT-306 x G.Til-10 & -3.20 & $38.40^{* * *}$ & $-30.80 * *$ & $-20.00^{* *}$ & 10.08 & $39.08^{* *}$ & $65.55^{* * *}$ & $24.46^{* * *}$ & 5.26 & $34.97^{* * *}$ & $18.63 * *$ & $29.59 * *$ & 3.59 & -6.54 & $12.79^{*}$ \\
\hline 12 & AT-306 x RT-54 & $-60.11^{* * *}$ & $-38.80^{* * *}$ & $-59.02 * *$ & $-49.73 * *$ & $-55.27 * *$ & $-58.05^{* *}$ & $-46.41^{* *}$ & $-46.04 * *$ & $-51.58^{* *}$ & $-50.56^{* *}$ & $-64.22^{* * *}$ & $-58.05 * *$ & $-55.09^{* *}$ & $-57.01 * *$ & $-58.69 * *$ \\
\hline 13 & AT-307 x AT-285 & $-32.29 * *$ & $53.65^{* * *}$ & $-41.67 * *$ & 2.08 & $31.13^{* *}$ & $-25.29 * *$ & $41.15^{* * *}$ & $-19.42^{*}$ & 3.16 & 2.95 & $-36.27^{* * *}$ & 10.49 & $-32.93^{* * *}$ & -8.41 & $-13.97^{*}$ \\
\hline 14 & AT-307 x G.Til-1 & $-41.15^{* * *}$ & -2.60 & $-50.52 * *$ & $-21.35^{* *}$ & -10.20 & $-35.06 * *$ & -10.53 & $-31.65^{* * *}$ & $-20.53^{* *}$ & $-23.31^{* *}$ & $-44.61^{* * *}$ & $-29.96 * *$ & $-43.11^{* *}$ & $-29.44 * *$ & $-35.92 * *$ \\
\hline 15 & AT-307 x G.Til-10 & $-50.40^{* * *}$ & $-24.0^{* * *}$ & $-53.20 * *$ & $-38.00^{* *}$ & $-32.88^{* *}$ & $-28.74 * *$ & -9.09 & -15.83 & $-18.42^{* *}$ & $-17.70^{*}$ & $-39.22^{* *}$ & $-28.84 * *$ & $-29.94 * *$ & $-27.57 * *$ & $-31.22 * *$ \\
\hline 16 & AT-307 x RT-54 & $-15.10^{*}$ & $21.88 * *$ & $-48.44 * *$ & $-37.50 * *$ & $-21.73^{* *}$ & -6.32 & 11.96 & $-28.78 * *$ & $-36.84 * *$ & -13.48 & $-20.10^{* * *}$ & $-12.36^{*}$ & $-40.72 * *$ & $-43.93 * *$ & $-27.70^{* *}$ \\
\hline 17 & AT-319 x AT-285 & $46.15 * *$ & $95.10 * *$ & -0.70 & $23.78^{* *}$ & $56.40 * *$ & $20.11 *$ & $33.49 * *$ & 2.16 & -6.84 & 13.34 & 2.45 & 4.49 & $-14.97 *$ & $-17.29 * *$ & -5.28 \\
\hline 18 & AT-319 x G.Til-1 & $-37.09 * *$ & 8.61 & $-38.41 * *$ & -10.60 & $-19.90 *$ & $-45.40^{* * *}$ & $-21.53 * *$ & $-33.09 * *$ & $-28.95 * *$ & $-31.60^{* * *}$ & $-53.43 * *$ & $-38.58 * *$ & $-44.31 * *$ & $-36.92 * *$ & $-42.84 * *$ \\
\hline 19 & AT-319 x G.Til-10 & 14.40 ** & $82.80 * *$ & $-36.00^{* * *}$ & $-29.20 * *$ & $23.71 * *$ & $64.37 * *$ & $118.66 * *$ & 15.11 & -6.84 & $51.69^{* * *}$ & $40.20^{\text {*** }}$ & $71.16 * *$ & -4.19 & $-17.29 * *$ & $26.76 * *$ \\
\hline 20 & AT- $319 \times$ RT -54 & $-45.90 * *$ & $-18.58^{*}$ & $-69.95 * *$ & $-18.58 * *$ & $-42.57 * *$ & $-43.10^{* *}$ & $-28.71 * *$ & $-60.43 * *$ & $-21.58 * *$ & $-36.52^{* * *}$ & $-51.47^{* * *}$ & $-44.19 * *$ & $-67.07 * *$ & $-30.37 * *$ & $-46.95 * *$ \\
\hline 21 & AT-322 $\times$ AT-285 & -18.88 & $91.61^{* *}$ & -9.09 & $80.42 * *$ & $51.07^{* *}$ & $-33.33^{* *}$ & $31.10^{* *}$ & -6.47 & $35.79^{* *}$ & 9.27 & $-43.14 * *$ & 2.62 & $-22.16^{* *}$ & $20.56^{* *}$ & -8.69 \\
\hline 22 & AT-322 x G.Til-1 & $-52.98 * *$ & $-35.76 * *$ & $-25.83 * *$ & 5.30 & $-27.80 * *$ & $-59.20 * *$ & $-53.59 * *$ & $-19.42 *$ & $-16.32 *$ & $-38.34^{* * *}$ & -65.20 ** & $-63.67 * *$ & $-32.93 * *$ & $-25.70 * *$ & $-48.47 * *$ \\
\hline 23 & AT- $322 \times$ G.Til- 10 & -2.00 & $67.20^{* *}$ & $-37.20^{* * *}$ & -9.20 & $19.93 * *$ & $40.80^{* *}$ & $100.00^{* * *}$ & 12.95 & $19.47 * *$ & $47.05^{* * *}$ & $20.10^{* * *}$ & $56.55 * *$ & -5.99 & 6.07 & $22.89^{*} *$ \\
\hline 24 & AT- $322 \times$ RT-54 & $-55.19^{* * *}$ & -10.38 & $-38.80^{* * *}$ & $-57.92 * *$ & $-44.73^{* *}$ & $-52.87 * *$ & $-21.53^{* * *}$ & $-19.42 *$ & $-59.47 * *$ & $-38.90^{* * *}$ & $-59.80^{* * *}$ & $-38.58 * *$ & $-32.93 * *$ & $-64.02 * *$ & $-48.94 * *$ \\
\hline 25 & AT-341 x AT-285 & $-35.14 * *$ & $-37.45 * *$ & $-44.40^{* * *}$ & $-33.20^{* * *}$ & $-31.68 * *$ & -3.45 & $-22.49 * *$ & 3.60 & -8.95 & -9.13 & $-17.65^{* * *}$ & $-39.33 * *$ & -13.77 & $-19.16^{* *}$ & $-24.06 * *$ \\
\hline 26 & AT-341 x G.Til-1 & $-36.68 * *$ & $-31.66 * *$ & $-46.33^{* * *}$ & $-49.81 * *$ & $-35.59 * *$ & -5.75 & $-15.31 *$ & 0.00 & $-31.58 * *$ & -14.33 & $-19.61 * *$ & $-33.71 * *$ & $-16.77 *$ & $-39.25 * *$ & $-28.40 * *$ \\
\hline 27 & AT-341 x G.Til-10 & $11.20^{*}$ & $37.45^{* *}$ & $-28.19^{* * *}$ & -9.65 & $12.35^{*}$ & $65.52^{* * *}$ & $70.33^{* * *}$ & $33.81^{* *}$ & $23.16^{* *}$ & $49.44^{* * *}$ & $41.18^{* * *}$ & $33.33^{* *}$ & 11.38 & 9.35 & $24.88^{* *}$ \\
\hline 28 & AT- $341 \times$ RT-54 & $-47.88 * *$ & $-22.01 * *$ & $-62.93 * *$ & $-40.15^{* *}$ & $-37.91 * *$ & $-22.41 * *$ & -3.35 & $-30.94 * *$ & $-18.42 * *$ & $-17.42 *$ & $-33.82 * *$ & $-24.34 * *$ & $-42.51 * *$ & $-27.57 * *$ & $-30.99 * *$ \\
\hline 29 & Bhuva-2 $\mathrm{x}$ AT-285 & $19.23^{*}$ & 13.46 & $-28.85^{* * *}$ & $-28.21 * *$ & -2.33 & 6.90 & $-15.31^{*}$ & $-20.14 *$ & $-41.05 * *$ & $-17.70^{*}$ & -8.82 & $-33.71 * *$ & $-33.53 * *$ & $-47.66^{* * *}$ & $-31.22 * *$ \\
\hline 30 & Bhuva-2 x G.Til-1 & -9.62 & -7.69 & 4.49 & 1.92 & -0.16 & $-18.97^{*}$ & $-31.10^{* *}$ & $17.27 *$ & $-16.32^{*}$ & -14.75 & $-30.88 * *$ & $-46.07 * *$ & -2.40 & $-25.70^{* *}$ & $-28.76^{* * *}$ \\
\hline 31 & Bhuva-2 $\times$ G.Til- 10 & $-18.80^{* * *}$ & $-15.60^{*}$ & $-32.00^{* * *}$ & $-22.00^{* *}$ & -10.77 & $16.67^{*}$ & 0.96 & $22.30^{*}$ & 2.63 & 9.41 & -0.49 & $-20.97 * *$ & 1.80 & -8.88 & -8.57 \\
\hline 32 & Bhuva-2 x RT-54 & $-43.17 * *$ & $-16.94 *$ & $-63.93 * *$ & $-36.07 * *$ & $-44.22 * *$ & $-40.23 * *$ & $-27.27^{* * *}$ & $-52.52 * *$ & $-38.42 * *$ & $-38.34 * *$ & $-49.02 * *$ & $-43.07 * *$ & $-60.48 * *$ & $-45.33 * *$ & $-48.47 * *$ \\
\hline 33 & Khadkala-S xAT-285 & $-30.63 * *$ & $-21.25 *$ & $-37.50^{* * *}$ & -15.00 & -15.84 & $-36.21 * *$ & $-39.71 * *$ & $-28.06^{* * *}$ & $-28.42 * *$ & $-33.57^{* * *}$ & $-45.59 * *$ & $-52.81 * *$ & $-40.12 * *$ & $-36.45 * *$ & $-44.48 * *$ \\
\hline 34 & Khadkala-S x GTil-1 & -3.75 & $65.63 * *$ & -10.63 & $-23.75 * *$ & 12.50 & -11.49 & $26.79 * *$ & 2.88 & $-35.79 * *$ & -3.93 & $-24.51 * *$ & -0.75 & $-14.37^{*}$ & $-42.99 * *$ & $-19.72 * *$ \\
\hline 35 & Khadkala-S xG.Til10 & $-39.60 * *$ & -1.20 & $-50.00^{* * *}$ & $-54.80^{* * *}$ & $-27.15^{* *}$ & -13.22 & $18.18^{*}$ & -10.07 & $-40.53 * *$ & -10.67 & -25.98 ** & -7.49 & $-25.15 * *$ & $-47.20^{* * *}$ & $-25.35 * *$ \\
\hline 36 & Khadkala-S x RT-54 & $-52.46 * *$ & $-56.83 * *$ & $-32.24 * *$ & -44.26 ** & $-50.19^{* *}$ & $-50.00^{* *}$ & $-62.20 * *$ & -10.79 & $-46.32 * *$ & $-44.94 * *$ & -57.35 ** & $-70.41 * *$ & $-25.75 * *$ & $-52.34 * *$ & $-53.99 * *$ \\
\hline 37 & IS-209 x AT-285 & $-36.3 * *$ & $-39.04 * *$ & $-52.74 * *$ & $-36.30^{* * *}$ & $-40.59 * *$ & $-46.55^{* * *}$ & $-57.42 * *$ & $-50.36^{* * *}$ & $-51.05 * *$ & $-51.69^{* * *}$ & -54.41 ** & $-66.67 * *$ & $-58.68 * *$ & $-56.54 * *$ & $-59.62 * *$ \\
\hline 38 & IS-209 x G.Til-1 & -11.92 & $20.53^{*}$ & $-16.56^{*}$ & 11.26 & 0.160 & $-23.56 * *$ & -12.92 & -9.35 & -11.58 & -14.47 & $-34.80^{* * *}$ & $-31.84 * *$ & $-24.55 * *$ & $-21.50^{* * *}$ & $-28.52 * *$ \\
\hline 39 & IS-209 x G.Til-10 & -8.80 & 4.40 & $-48.40^{* *}$ & $-10.40^{*}$ & -3.55 & $31.03 * *$ & $24.88^{* * *}$ & -7.19 & $17.89^{* * *}$ & $18.26^{*}$ & 11.76 & -2.25 & $-22.75 * *$ & 4.67 & -1.17 \\
\hline \multirow{4}{*}{40} & IS-209 x RT-54 & $-45.36^{* * *}$ & $-37.16^{* *}$ & $-38.8^{* * *}$ & $-40.44 * *$ & $-44.60^{* * *}$ & $-42.53^{* *}$ & $-44.98^{* *}$ & $-19.42^{*}$ & $-42.63^{* * *}$ & $-38.76^{* * *}$ & $-50.98 * *$ & $-56.93 * *$ & $-32.93 * *$ & $-49.07 * *$ & $-48.83^{* *}$ \\
\hline & Min. & -60.11 & -56.83 & -69.95 & -57.92 & -55.27 & -59.20 & -62.20 & -60.43 & -59.47 & -51.69 & -65.20 & -70.41 & -67.07 & -64.02 & -59.62 \\
\hline & Max. & 46.15 & 95.10 & 4.49 & 80.42 & 56.40 & 65.52 & 118.66 & 33.81 & 35.79 & 51.69 & 41.18 & 71.16 & 11.38 & 20.56 & 26.76 \\
\hline & Mean heterosis & -21.72 & 10.56 & -35.75 & -18.61 & -10.74 & -13.06 & 1.84 & -13.26 & -20.25 & -10.64 & -25.85 & -20.28 & -27.80 & -29.19 & -25.33 \\
\hline Desir & heterosis & 5 & 15 & 0 & 4 & 7 & 10 & 13 & 5 & 4 & 7 & 7 & 6 & 0 & 1 & 4 \\
\hline
\end{tabular}


Table.7 Ten most heterobeltiosis $\left(\mathrm{H}_{1}\right)$ crosses along with standard heterosis $\left(\mathrm{H}_{2}\right)$ and per se performance for seed yield and traits over environments in sesame

\begin{tabular}{|c|c|c|c|c|c|c|c|c|c|c|c|c|c|c|c|c|c|}
\hline \multirow[b]{2}{*}{$\begin{array}{l}\text { Sr. } \\
\text { No }\end{array}$} & \multirow{2}{*}{ Crosses } & \multirow{2}{*}{$\begin{array}{l}\text { Mean } \\
\text { seed } \\
\text { yield } \\
\text { /plant } \\
\text { (g) }\end{array}$} & \multicolumn{3}{|c|}{ Seed yield per plant (g) } & \multicolumn{3}{|c|}{ Day to flowering } & \multicolumn{3}{|c|}{ Days to maturity } & \multicolumn{3}{|c|}{ Plant height (cm) } & \multicolumn{3}{|c|}{ Height to first capsule } \\
\hline & & & $\mathbf{H}_{1}$ & $\mathrm{H}_{2} \mathrm{C}_{1}$ & $\mathrm{H}_{2} \mathrm{C}_{2}$ & $\mathbf{H}_{1}$ & $\mathrm{H}_{2} \mathrm{C}_{1}$ & $\mathbf{H}_{2} \mathbf{C}_{2}$ & $\mathbf{H}_{1}$ & $\mathbf{H}_{2} \mathbf{C}_{1}$ & $\mathbf{H}_{2} \mathbf{C}_{2}$ & $\mathbf{H}_{1}$ & $\mathrm{H}_{2} \mathrm{C}_{1}$ & $\mathbf{H}_{2} \mathbf{C}_{2}$ & $\mathbf{H}_{1}$ & $\mathrm{H}_{2} \mathrm{C}_{1}$ & $\mathrm{H}_{2} \mathrm{C}_{2}$ \\
\hline 1 & AT-319 x AT-285 & 6.73 & $56.40 * *$ & 13.34 & -5.28 & 2.22 & -0.86 & 0.00 & -0.20 & -2.17 & -1.39 & 9.33 & $-8.32 *$ & $-3.70 * *$ & $26.07 * *$ & -4.42 & 6.60 \\
\hline 2 & AT-322 x AT-285 & 6.48 & $51.07 * *$ & 9.27 & -8.69 & -4.06 & -3.23 & -2.39 & -0.91 & -2.86 & -2.09 & $-7.26 *$ & $-15.81 * *$ & $-11.57 * *$ & -4.18 & $-20.06^{* *}$ & -10.84 \\
\hline 3 & AT-307 x AT-285 & 6.11 & $31.13 * *$ & 2.95 & $-13.97 *$ & -3.85 & -3.02 & -2.17 & 0.40 & -1.58 & -0.80 & $10.60 * *$ & -6.02 & -1.28 & $33.26 * *$ & -0.03 & 11.50 \\
\hline 4 & AT-319 x G.Til-10 & 9.00 & $23.71 * *$ & $51.69 * *$ & $26.76^{* *}$ & $5.56^{*}$ & 2.37 & 3.26 & -2.37 & 1.68 & 2.49 & 13.90 & -4.53 & 0.29 & $18.69^{*}$ & -10.01 & 0.36 \\
\hline 5 & AT-306 x AT-285 & 5.20 & 21.17 & -12.36 & $-26.76 * *$ & -4.27 & -3.45 & -2.61 & -2.92 & $-4.84 * *$ & $-4.08 *$ & 3.91 & $-8.02 * *$ & -3.39 & 12.09 & $-11.74 *$ & -1.57 \\
\hline 6 & AT-322 x G.Til-10 & 8.73 & $19.93 * *$ & $47.05 * *$ & $22.89 * *$ & -2.21 & 4.96 & $5.87 *$ & -1.43 & 1.88 & 2.69 & 5.91 & -3.85 & 0.99 & 8.07 & -9.84 & 0.56 \\
\hline 7 & $\begin{array}{c}\text { Khadakala x G.Til- } \\
1\end{array}$ & 5.70 & $12.50^{*}$ & -3.93 & $-19.72 * *$ & 2.70 & -1.51 & -0.65 & -0.69 & -1.09 & -0.30 & 6.31 & $-9.04 * *$ & -4.46 & 0.87 & -18.90 & -9.54 \\
\hline 8 & AT-341 x G.Til-10 & 8.87 & $12.35^{*}$ & $49.44 * *$ & $24.88 * *$ & 3.04 & 2.16 & 3.04 & 0.59 & 0.20 & 1.00 & -2.97 & $11.68 * *$ & $17.30 * *$ & $-22.66 * *$ & 10.01 & $22.70 * *$ \\
\hline 9 & AT-306 x G.Til-10 & 8.01 & 10.08 & $34.97 * *$ & $12.79 *$ & -2.37 & $6.47 *$ & $7.39 * *$ & 2.34 & 3.46 & 4.28 & $27.50 * *$ & $12.90 * *$ & $18.50 * *$ & $49.87 * *$ & $18.01 * *$ & $31.61 * *$ \\
\hline 10 & AT-253 x AT-285 & 5.28 & 8.39 & -11.10 & $-25.70 * *$ & -1.28 & -0.43 & $0.43^{* *}$ & 2.01 & 0.00 & 0.80 & -1.04 & $-18.2 * *$ & $-14.10^{* * *}$ & -14.86 & $-37.25^{* *}$ & $-30.01 * *$ \\
\hline
\end{tabular}


Likewise, for height to first capsule (Table 5) the range for heterobeltiosis in the individual environments i.e., $\mathrm{E}_{1}, \mathrm{E}_{2}, \mathrm{E}_{3}, \mathrm{E}_{4}$ and on pooled basis were -47.71 to $114.33 \%,-13.44$ to $256.18 \%,-48.12$ to $104.61 \%,-4.03$ to 241.85 and -22.66 to $88.70 \%$, respectively. Lowest height to first capsule over better parent was AT-341 x G.Til-10 followed by AT-341 x AT-285, Khadkala-S x G.Til-10, AT-253 x AT-285 and Khadkala-S x AT-285 in pooled analysis. Out of 40 hybrids, significant and negative heterobeltiosis crosses was recorded in $16,1,16,0$ and 4 in $E_{1}, E_{2}, E_{3}, E_{4}$ and across the environments, respectively. Out of 40 crosses, 19 crosses over G.Til-2 and 13 crosses over G.Til-3 exhibited significant and desirable (negative) standard heterosis across the environments. The range of standard heterosis in pooled over $\mathrm{H}_{2} \mathrm{C}_{1}$ and $\mathrm{H}_{2} \mathrm{C}_{2}$ was 37.25 to $25.15 \%$ and -30.01 to $39.58 \%$, respectively. The cross, AT-253 x AT-285 exhibited highest, negative and significance standard heterosis for $\mathrm{H}_{2} \mathrm{C}_{1}$ and $\mathrm{H}_{2} \mathrm{C}_{2}$ in pooled analysis.

For seed yield per plant $(\mathrm{g})$ heterobeltiosis (Table 6) ranged AT-306 x AT-285, AT-319 $x$ G.Til-10 and AT-253 x RT-54 in $\mathrm{E}_{1}$; AT319x G.Til-10, AT-322 x G.Til-10 and AT265 x G.Til-10 in E2; AT-341 x G.Til-10, AT306 x G.Til-10 and Bhuva-2 x G.Til-10 in $\mathrm{E}_{3}$ : AT-322 x AT-285, AT-341 x G.Til-10 and AT-322 x G.Til-10 in $E_{4}$. In pooled analysis, crosses, AT-319 x G.Til-10, AT-341 x G.Til10, AT-322 x G.Til-10, AT-306 x G.Til-10 and AT-253 x G.Til-10 exhibited significant and positive heterobeltiosis for high seed yield per plant.

Highest standard heterosis for $\mathrm{H}_{2} \mathrm{C}_{1}$ in seed yield per plant $(\mathrm{g})$ was recorded in AT-341 $\mathrm{x}$ G.Til-10 in $\mathrm{E}_{1}$, AT-319 x G.Til-10 in $\mathrm{E}_{2}$, AT341 x G.Til-10 in $E_{3}$, AT-322 x AT-285 in $E_{4}$ and AT-319 x G.Til-10 in pooled analysis. While, the values for $\mathrm{H}_{2} \mathrm{C}_{2}$ in seed yield per plant (g) was AT-341 x G.Til-10 in $\mathrm{E}_{1}$, AT-
$319 \times$ G.Til-10 in $E_{2}$, AT-341 x G.Til-10 in $\mathrm{E}_{3}, \mathrm{AT}-322 \times \mathrm{AT}-285$ in $\mathrm{E}_{4}$ and AT-319 $\mathrm{x}$ G.Til-10 in pooled analysis. The cross AT319 x G.Til-10 recorded maximum, significant and positive heterosis of $51.69 \%$ and $26.76 \%$ over both the checks across the environments. Crosses exhibiting significant and positive standard heterosis over G.Til-2 were $10,13,5,4$ and 7 as well as 7, 6, 0, 1 and 4 crosses recorded significant and positive heterosis over G.Til-3 in $\mathrm{E}_{1}, \mathrm{E}_{2}, \mathrm{E}_{3}$, $\mathrm{E}_{4}$ and across the environments, respectively.

The crosses exhibiting the highest ten significant positive heterobeltiosis and mean seed yield/plant $(\mathrm{g}$ ) for seed yield per plant were (Table 7) AT-319 x AT-285, AT-322 x AT-285, AT-307 x AT-285, AT-319 x G.Til10, AT-306 x AT-285, AT-322 x G.Til-10, Khadakala x G.Til-1, AT-341 x G.Til-10, AT306 x G.Til-10, AT-253 x AT-285 in pooled analysis. The results indicated that the degree and magnitude of heterosis varied from cross to cross for various characters under individual environments and in pooled analysis. Inconsistent performance of most of the hybrids across the environments for various characters suggested that parental genes and their combinations were sensitivities to environmental variation, which is the general feature of quantitative inheritance.

While considering the performance of hybrids over the environments in respect of seed yield per plant, seven hybrids over better parent and seven hybrids over standard check G.Til-2 and four hybrids over G.Til-3 exhibited significant and positive heterosis. The range of heterosis over better parent was from 55.27 to 56.40, while over standard check G.Til-2 and G.Til-3 it ranged from -51.69 to 51.69 and -59.62 to 26.76 , respectively. High heterosis for seed yield seed yield per plant has been reported by Monpara and Pawar (2016), Nayak et al., (2017), Virani et al., 
(2017), Chaudhary et al., (2018) and Karande et al., (2018).Highly significant and desirable heterobeltiosis was exhibited for plant height by AT-306 x G.Til-10 and AT-307 x AT-285; for height to first capsule by AT-341 x G.Til10 with seed yield per plant.

Hagberg (1952) observed similar effects and termed it "combinational heterosis". In order to see whether similar situation exist in sesame or not, a comparison of ten most heterotic crosses for seed yield was made with other yield related characters along with average mean seed yield per plant over environments (Table 8). The crosses AT-322 x AT-285, AT-307 x AT-285, AT-306 x AT285, and AT-253 x AT-285 which manifested significant and desirable heterobeltiosis and standard heterosis in earliness with seed yield per plant. Therefore, heterotic effects for seed yield per plant could be a result of combinational heterosis. Hence, to obtain maximum heterotic effects for seed yield per plant, desired level of heterosis of each component character should be worked-out to identify superior hybrids.

Overall the most heterotic crosses identified included AT-319 x AT-285, AT-253 x RT-54 and Bhuva-2 $x$ AT-285 in $\mathrm{E}_{1}$; AT-319 x AT285, AT-322 x AT-285 and AT-319 x G.Til10 in $\mathrm{E}_{2}$; Bhuva- $2 \times$ G.Til-1, AT-306 $x$ AT285 and AT-319 $x$ AT-285 in $\mathrm{E}_{3}$ and AT-322 $x$ AT-285, AT-319 x AT-285 and AT-253 x AT-285 in $\mathrm{E}_{4}$. In pooled analysis, the crosses AT-319 x AT-285, AT-322 x AT-285, AT$307 \times$ AT-285, AT-319 x G.Til-10 and AT$306 \times$ AT-285 exhibited significant and positive heterobeltiosis for seed yield per plant and its components. The crosses AT-322 x AT-285, AT-307 x AT-285, AT-306 x AT285, and AT-253 x AT-285 which manifested significant and desirable heterobeltiosis and standard heterosis in earliness with seed yield per plant. Such crosses could be exploited further for seed yield advancement in sesame through regional multilocational testing over different environments.

\section{References}

Allard, R. W. and Bradshaw, A. D. (1964). Implications of genotype environmental interactions in applied plant breeding. Crop Sci., 4: 503-508.

Anonymous, (2016). Status Paper on Oilseeds. Oilseeds Division, Department of Agriculture and Cooperation, Ministry of Agriculture, Government of India, New Delhi.

Bedigian, D. and Harlan, J. R. (1986). Evidence for cultivation of sesame in the ancient world. Econ Bot., 40 (2):137-154.

Bhat, K.V., Babrekar, P.P and Lakhanpaul. S. (1999). Study of genetic diversity in Indian and exotic sesame (Sesamum indicum L.) germplasm using random amplified olymorphic DNA (RAPD) markers. Euphytica., 110:21-33.

Chaudhary, B. K., Solanki, S. D., Singh, S., Prajapati, N. N. and Chaudhary, V. B. (2018). Heterosis studies for seed yield and its components in sesame. (Sesamum indicum L.). Int. J. Agri. Sci., 10 (5): 5383 - 5386.

Fonseca, S. and Patterson, F.C. (1968). Hybrid vigour in seven parent diallel in common winter wheat (Triticum aestivum L.). Crop Sci., 8: 85-88.

Hagberg, A. (1952). Heterosis in F1 combinations in Galeopsis-I and II. Heriditas., Lund. 1: 221-225. (Fide: Indian J. Genet., 29 (1): 53-61.

Jones, D.F. (1917). Dominance of linked factors as a means of accounting for heterosis. Genetics., 2: 466-479.

Karande, G. R., Yamgar1, S.V., Waghmode, A. A. and Wadikar, P. B. (2018). Exploitation of heterosis for yield and yield contributing character in Sesame 
(Sesamum indicum L.). Int. J. Curr. Microbiol. App. Sci., 7(2):299-308.

Mather, K. (1943). Polygenic inheritance and natural selection. Biol. Rev., 18: 32-In: $\mathrm{G} \times \mathrm{E}$ interaction, its measurement and significance in Plant Breeding. (Eds. Verma, M.M. and Gill, K.S.). PAU Bull., 1: 7-18.

Monpara, B. A. and Pawar A. K. (2016). Evaluation of hybrids for heterosis breeding in sesame (Sesamum indicum L.). Elec. J. Pl. Breed., 7 (4): 11831187.

Najeeb, U., Mirza, M. Y., Jilani, G., Mubashir, A. K. and Zhou, W. J. (2012) Sesame. In: Gupta SK (ed), Technological innovations in major world oil crops, vol 1. Springer, New York, pp 131-145.

Nayak, A. J., Patel, S. R. and Shrivastva, A. (2017). Heterosis studies for yield and its components traits in sesame (Sesamum indicum L.). AGRES - An Int. J., 6 (1): 38-48.
Panse, V.G. and Sukhatme, P.V. (1985). Statistical Methods for Agricultural Workers. Indian Council of Agricultural Research, New Delhi.

Paroda, R.S. and Hayes, J.D. (1971). An investigation of genotype - environment interactions on rate of ear emergence in spring barley. Heredity., 26: 157-175.

Samuel, C. J. A., Hill, J., Breese, E.L. and Davis, A. (1970). Assessing and predicting environmental response in Lolium Pereene J. Agric. Sci. Comb., 75: 1-9.

Singh, M. and Singh, R. K. (1984). A comparison of different methods of half diallel analysis. Theor. Appl. Genet., 67: 323-326.

Virani, M. B., Vachhani, J. H., Kachhadia. V. H., Chavadhari, R. M. and Mungala R. A. (2017). Heterosis studies in sesame (Sesamum indicum L.), Elec. J. Pl. Breed., 8 (3): 1006-1012.

Weiss, E. A., (1983). Oilseed Crops, Longman, New York.

\section{How to cite this article:}

Parmar, R.S., V.P. Chovatia, G.K. Sapara and Dhingani, J.C. 2018. Breeding for Earliness and Seed Yield in Summer Sesame (Sesamum indicum L.) Over Environments. Int.J.Curr.Microbiol.App.Sci. 7(10): 1523-1536. doi: https://doi.org/10.20546/ijcmas.2018.710.170 